TRANSACTIONS OF THE

AMERICAN MATHEMATICAL SOCIETY

Volume 349, Number 1, January 1997, Pages 129-153

S 0002-9947(97)01825-4

\title{
INTEGRATION OF CORRESPONDENCES ON LOEB SPACES
}

\author{
YENENG SUN
}

\begin{abstract}
We study the Bochner and Gel'fand integration of Banach space valued correspondences on a general Loeb space. Though it is well known that the Lyapunov type result on the compactness and convexity of the integral of a correspondence and the Fatou type result on the preservation of upper semicontinuity by integration are in general not valid in the setting of an infinite dimensional space, we show that exact versions of these two results hold in the case we study. We also note that our results on a hyperfinite Loeb space capture the nature of the corresponding asymptotic results for the large finite case; but the unit Lebesgue interval fails to provide such a framework.
\end{abstract}

\section{INTRODUCTION}

Since Aumann's seminal work [6] on the integration of a finite dimensional valued correspondence, there have been numerous applications and generalizations of that work. See [4, 12, 20, 29], and [32] for some of the results. The extension of Aumann's theory to an infinite dimensional space has also been studied extensively by many authors since the pioneering work of Debreu [12] in that direction. For references, see $[7,10,19,26,35]$, and [46]. Here we note that in the literature correspondences are also referred to as multifunctions, set valued functions, set valued maps, and random sets.

Two results in [6] are crucial for applications. The first is the Lyapunov type result on the compactness and convexity of the integral of a finite dimensional valued correspondence on an atomless measure space. The second is the Fatou type result on the preservation of upper semicontinuity by integration. It is well known that those two results fail in the setting of an infinite dimensional space for some correspondences on the unit Lebesgue interval (see [13], and also [38, 46]).

The aim of this paper is to note that if instead of working on a general measure space, we consider Banach space valued correspondences on a general Loeb space, then we can obtain exact versions of both Fatou type and Lyapunov type results for Bochner and Gel'fand integrals of those correspondences, which will enable one to apply a fixed point argument to solve relevant problems (see [8, 15], and [16]

Received by the editors February 23, 1995.

1991 Mathematics Subject Classification. Primary 03H05, 28B20; Secondary 46G10, 90A14.

Key words and phrases. Correspondences, Loeb spaces, Bochner integral, Gel'fand integral, convexity, semicontinuity, weak compactness, weak* compactness .

The main results were presented at the Fifth Asian Logic Conference held in Singapore in June 1993. The author is grateful to Professors Robert Anderson, Donald Burkholder, Chi Tat Chong, Ward Henson, Zhuxin Hu, Jerome Keisler, Ali Khan, Peter Loeb, Walter Trockel, and Jerry Uhl for helpful conversations and encouragement. The research is partially supported by the National University of Singapore, grant no. RP3920641. 
for fixed point theorems in an infinite dimensional space). Moreover, once we have results on a hyperfinite Loeb space, the usual techniques of lifting, pushing-down, and transfer (see $[1,2,31,36]$ ) allow us to obtain results for the large finite case (see, for example, Proposition 4.11 below). Note that people usually use Lebesgue spaces or more specifically the unit Lebesgue interval as an idealization of a large finite model (see [5] and [20]). Since approximate results are usually more difficult to find for a finite model, they also try to obtain asymptotic results for a large finite model by finding exact results for the ideal model first (see [20]). Thus the failure of the Lyapunov and Fatou type results in the setting of an infinite dimensional space indicates that the Lebesgue spaces fail to provide such a framework for a large class of problems. Here we also note that many infinite models in scientific study are idealizations of finite but large number of objects in reality. Thus those infinite models which capture the nature of the corresponding large finite models should have many advantages over other infinite models.

In [43], we have developed a distributional theory for correspondences on a Loeb space. We shall use some of the results shown there to prove the main results of this paper. In joint work with Ali Khan (see [27] and [28]), the results in this paper and [43] are systematically used to study relevant problems in game theory and general equilibrium theory. Here we note that the distribution theory is also related to the integration theory in the following way. The distribution of a measurable function $f$ from a probability space $(T, \mathcal{T}, \nu)$ to a compact metric space $X$ is the Gel'fand integral of the mapping $f_{\delta}$ from $(T, \mathcal{T}, \nu)$ to the space of Borel measures on $X$ which is the dual space of the space of continuous functions on $X$, where for each $t \in T$, $f_{\delta}(t)$ is the Dirac measure on $X$ concentrated at the point $f(t)$.

The paper is organized as follows. In Section 2 we state the main results. In Section 3 we collect some results in [43] which are needed in this paper. The final two sections are devoted respectively to the proof of the main results on the Bochner and Gel'fand integrals of Banach space valued correspondences.

\section{The MAIN RESULTS}

We first introduce some basic definitions. Let $T$ and $S$ be nonempty sets, and $\mathcal{P}(S)$ the power set of $S$. A mapping from $T$ to $\mathcal{P}(S)-\{\emptyset\}$ is called a correspondence from $T$ to $S$. Let $X$ be a topological space, $\mathcal{B}(X)$ the Borel $\sigma$-algebra of $X$, and $F$ a correspondence from a probability space $(T, \mathcal{T}, \nu)$ to $X$, where $\mathcal{T}$ is a $\sigma$-algebra on $T$ and $\nu$ a probability measure on $(T, \mathcal{T})$. For simplicity, in this paper we shall only work with probability measures which are complete on the corresponding measurable spaces. It is also obvious that the results reported in this paper can be restated for a finite measure space. The correspondence $F$ is said to be measurable if its graph $\{(t, x) \in T \times X: x \in F(t)\}$ belongs to the product $\sigma$-algebra $\mathcal{T} \otimes \mathcal{B}(X)$. See Chapter III of [11] for many equivalent definitions of the measurability of a correspondence. A function $f$ from $(T, \mathcal{T}, \nu)$ to $X$ is called a selection of $F$ if $f(t) \in F(t)$ for all $t \in T$. If, in addition, $f$ is measurable, then $f$ is said to be a measurable selection. Note that if $F$ is measurable, then $F$ has a measurable selection (see [20, p. 54] and also [44] for a survey of measurable selections). $F$ is said to be closed valued if $F(t)$ is a closed subset of $X$ for all $t \in T$. We can define compact valued correspondences similarly.

Now let $f$ be a function from a probability space $(T, \mathcal{T}, \nu)$ to a Banach space $X$. If $f$ can be approximated in norm by a sequence of simple functions and $\|f\|$ is 
integrable on the space $(T, \mathcal{T}, \nu)$, then $f$ is said to be Bochner integrable (see [13]). For a correspondence $F$ from $(T, \mathcal{T}, \nu)$ to $X$, let $\mathcal{S}_{F}$ be the collection of all Bochner integrable selections of $F$ and let the Bochner integral $\int_{T} F d \nu$ of the correspondence $F$ be the set $\left\{\int_{T} f d \nu: f \in \mathcal{S}_{F}\right\}$ in $X . F$ is said to be integrably bounded if there is a real valued integrable function $g$ on $(T, \mathcal{T}, \nu)$ such that for $\nu$-almost all $t \in T$, $\sup \{\|x\|: x \in F(t)\} \leq g(t)$. When $X$ is a finite dimensional space, by using the Lyapunov theorem on the range of an atomless vector measure, Richter [37] showed that $\int_{T} F d \nu$ is convex. Also Aumann showed in [6] that if $F$ is integrably bounded, then $\int_{T} F d \nu$ is compact. The following well known example of Lyapunov indicates that the Aumann-Richter results are not valid for an $l_{2}$-valued correspondence on the unit Lebesgue interval (see [13] and also [38, 46]).

Example 1. Let $([0,1], \mathcal{L}([0,1]), \lambda)$ be the unit Lebesgue interval, where $\mathcal{L}([0,1])$ is the the collection of Lebesgue measurable sets in $[0,1]$ and $\lambda$ is the Lebesgue measure. Select a complete orthogonal system $\left\{w_{n}\right\}_{n=0}^{\infty}$ in the space $L_{2}(\lambda)$ of real valued square integrable functions on $[0,1]$ such that each $w_{n}$ assumes only the values 1 or -1 and such that $w_{0}(t)=1$ for each $t \in[0,1]$ and $\int_{0}^{1} w_{n} d \lambda=0$ for each $n \geq 1$. Define a function $f$ from $[0,1]$ to $l_{2}$ by letting

$$
f(t)=\left(\frac{1+w_{n}(t)}{2^{n+1}}\right)_{n=0}^{\infty}
$$

for each $t \in[0,1]$. For each $n \geq 1$, let $U_{n}=\left\{t: w_{n}(t)=1\right\}$. It is clear that $f$ is Bochner integrable and

$$
\int_{[0,1]} f d \lambda=\left(1, \frac{1}{2^{2}}, \cdots, \frac{1}{2^{n+1}}, \cdots\right) .
$$

For each $t \in[0,1]$, let $F(t)$ be the set $\{0, f(t)\}$ in $l_{2}$. Then $F$ is a norm compact valued, integrably bounded, measurable correspondence. Suppose that $\int_{[0,1]} F d \lambda$ is convex. Then there is a Lebesgue measurable subset $E$ of $[0,1]$ such that

$$
\int_{E} f d \lambda=\frac{1}{2} \int_{[0,1]} f d \lambda=\left(\frac{1}{2}, \frac{1}{2^{3}}, \cdots, \frac{1}{2^{n+2}}, \cdots\right) .
$$

Thus $\lambda(E)=1 / 2$ and for each $n \geq 1$,

$$
\lambda\left(E \cap U_{n}\right)=\lambda\left(E-U_{n}\right)=\lambda\left(U_{n}-E\right)=\lambda\left([0,1]-\left(E \cup U_{n}\right)\right)=\frac{1}{4} .
$$

Define a function $h$ on $[0,1]$ such that $h(t)=1$ for $t \in E$ and $h(t)=-1$ for $t \in$ $[0,1]-E$. Then $h$ is orthogonal to all the $w_{n}$ 's, which contradicts the completeness of the system. Hence $\int_{[0,1]} F d \lambda$ is not convex.

Next for each $m \geq 1$, let $f_{m}(t)=\left(\frac{1+w_{n}(t)}{2^{n+1}}\right)_{n=0}^{m}$ and $F_{m}(t)=\left\{0, f_{m}(t)\right\} \subseteq$ $\mathbb{R}^{m+1}$. Then the Lyapunov theorem says that $\int_{[0,1]} F_{m} d \lambda$ is convex. Thus there is a Lebesgue measurable set $E_{m}$ in $[0,1]$ such that

$$
\int_{E_{m}} f_{m} d \lambda=\frac{1}{2} \int_{[0,1]} f_{m} d \lambda
$$

It is easy to see that $\int_{E_{m}} f d \lambda \in \int_{[0,1]} F d \lambda$ and $\lim _{m \rightarrow \infty} \int_{E_{m}} f_{m} d \lambda=\frac{1}{2} \int_{[0,1]} f d \lambda$. Thus $\int_{[0,1]} F d \lambda$ is not norm closed and hence not weakly closed. Therefore $\int_{[0,1]} F d \lambda$ is neither norm compact nor weakly compact. 
Next we consider correspondences on a Loeb space. Let $\Omega$ be a nonempty internal set, $\mathcal{A}$ an internal algebra of subsets of $\Omega$, and $P$ a finitely additive internal probability measure on $(\Omega, \mathcal{A})$. Define a real valued set function ${ }^{\circ} P$ on $(\Omega, \mathcal{A})$ such that for each $A \in \mathcal{A},{ }^{\circ} P(A)$ is the standard part ${ }^{\circ}(P(A))$ of $P(A)$. Then Loeb's theorem (see [31]) indicates that ${ }^{\circ} P$ can be extended to a probability measure $L(P)$ on the $\sigma$-algebra generated by $\mathcal{A}$. Let $(\Omega, L(\mathcal{A}), L(P))$ be the completion of the space $(\Omega, \sigma(\mathcal{A}), L(P))$, which is usually referred to as the Loeb space.

The first theorem says that the Bochner integral of any Banach space valued correspondence on any atomless Loeb space is always convex. Note that even though the result is completely general, i.e., neither measurability nor closedness on the correspondence is required, it is in fact almost the easiest to prove among the theorems presented in this section.

Theorem 1. Let $F$ be any correspondence from an arbitrarily given atomless Loeb space $(\Omega, L(\mathcal{A}), L(P))$ to any Banach space $X$. Then the Bochner integral $\int_{\Omega} F d L(P)$ of $F$ is always convex.

The next theorem concerns the norm compactness of the Bochner integral of a Banach space valued correspondence on a Loeb space. Note that the integrable boundedness of the correspondence is needed even if the Banach space is finite dimensional (see [6, p. 9]).

Theorem 2. Let $F$ be any norm compact valued correspondence from any given Loeb space $(\Omega, L(\mathcal{A}), L(P))$ to a general Banach space $X$. If $F$ is integrably bounded, then $\int_{\Omega} F d L(P)$ is norm compact.

Remark. Let $(T, \mathcal{T}, \nu)$ be a positive measure space, and $G$ a vector measure from $(T, \mathcal{T})$ to a Banach space $X$. Assume that $G$ has a Radon-Nikodým derivative $f$ with respect to $\nu$, i.e., there is a Bochner integrable function $f$ from $(T, \mathcal{T}, \nu)$ to $X$ such that $G(A)=\int_{A} f d \nu$ for any $A \in \mathcal{T}$. Then it is clear that the range of $G$ is $\int_{\Omega} F d \nu$, where $F$ is the correspondence defined by $F(t)=\{0, f(t)\}$. Hence the convexity or norm compactness of $\int_{\Omega} F d \nu$ would imply that of the range of $G$. Thus Theorems 1 and 2 imply that the range of any Banach space valued measure with a Radon-Nikodým derivative on any given atomless Loeb space must be convex and norm compact. That is, the original version of Lyapunov's theorem holds in this case.

Since norm compact sets are in a certain sense quite small in an infinite dimensional Banach space, weakly compact sets are often used in various applications. The following theorem is an analog of Theorem 2 in the setting of weakly compact sets.

Theorem 3. Let $F$ be any integrably bounded, weakly compact valued correspondence from $(\Omega, L(\mathcal{A}), L(P))$ to a general Banach space $X$. Then $\int_{\Omega} F d L(P)$ is weakly compact.

In the rest of this section, we shall only work with a general separable Banach space $X$. The following theorem is a version of Aumann's Theorem 3 in [6] in the setting of Banach spaces. Note that the result fails on the unit Lebesgue interval. The correspondence $F$ in Example 1 provides a counterexample.

Theorem 4. Let $F$ be a measurable, integrably bounded, weakly compact valued correspondence from a Loeb space $(\Omega, L(\mathcal{A}), L(P))$ to a separable Banach space $X$. 
If $(\Omega, L(\mathcal{A}), L(P))$ is atomless, then

$$
\int_{\Omega} \overline{c o} F d L(P)=\int_{\Omega} F d L(P),
$$

where $\overline{c o} F$ is the correspondence such that for each $\omega \in \Omega, \overline{c o} F(\omega)$ is the norm closure of the convex hull of $F(\omega)$.

Now we come to the property that integration preserves semicontinuity. The following definition of semicontinuity is adopted (see [4, pp. 38-40] or [20, pp. 21-26]).

Definition. Let $G$ be a correspondence from a topological space $Y$ to another topological space $Z$. Let $y_{0}$ be a point in $Y$. Then $G$ is said to be upper semicontinuous at $y_{0}$ if for any open set $U$ which contains $G\left(y_{0}\right)$, there exists a neighborhood $V$ of $y_{0}$ such that $y \in V$ implies that $G(y) \subseteq U$. $G$ is said to be lower semicontinuous at $y_{0}$ if for any open set $U$ with $U \cap G\left(y_{0}\right) \neq \emptyset$, there exists a neighborhood $V$ of $y_{0}$ such that $G(y) \cap U \neq \emptyset$ for every $y \in V$. $G$ is said to be continuous at $y_{0}$ if it is both upper and lower semicontinuous at $y_{0}$.

We still work on a Loeb probability space $(\Omega, L(\mathcal{A}), L(P))$. Let $Y$ be a metric space and $F$ a correspondence from $\Omega \times Y$ to a separable Banach space $X$. Assume that for each fixed $y \in Y, F(\cdot, y)$ is a measurable correspondence from $\Omega$ to $X$. Theorem 5 concerns norm semicontinuity and Theorem 6 involves semicontinuity in the weak topology.

Theorem 5. Assume that $F$ is norm closed valued and there is an integrably bounded, norm compact valued correspondence $G$ such that for every $y \in Y, F(\omega, y)$ $\subseteq G(\omega)$ for almost all $\omega \in \Omega$. Then if $F(\omega, y)$ is norm upper semicontinuous on $Y$ for each fixed $\omega$, then $\int_{\Omega} F(\omega, y) d L(P)(\omega)$ is norm upper semicontinuous on $Y$; if $F(\omega, y)$ is norm lower semicontinuous on $Y$ for each fixed $\omega$, then $\int_{\Omega} F(\omega, y) d L(P)(\omega)$ is norm lower semicontinuous on $Y$; if $F(\omega, y)$ is norm continuous on $Y$ for each fixed $\omega$, then $\int_{\Omega} F(\omega, y) d L(P)(\omega)$ is norm continuous on $Y$.

Theorem 6. Assume that $F$ is weakly closed valued and there is an integrably bounded, weakly compact valued correspondence $G$ such that for every $y \in Y$, $F(\omega, y) \subseteq G(\omega)$ for almost all $\omega \in \Omega$. Then if $F(\omega, y)$ is weakly upper semicontinuous on $Y$ for each fixed $\omega$, then $\int_{\Omega} F(\omega, y) d L(P)(\omega)$ is weakly upper semicontinuous on $Y$; if $F(\omega, y)$ is weakly lower semicontinuous on $Y$ for each fixed $\omega$, then $\int_{\Omega} F(\omega, y) d L(P)(\omega)$ is weakly lower semicontinuous on $Y$; if $F(\omega, y)$ is weakly continuous on $Y$ for each fixed $\omega$, then $\int_{\Omega} F(\omega, y) d L(P)(\omega)$ is weakly continuous on $Y$.

The following example shows that the results in the above two theorems on the preservation of upper semicontinuity by integration are not valid for some correspondence on the unit Lebesgue interval.

Example 2. We choose a system $\left\{w_{n}\right\}_{n=0}^{\infty}$ as in Example 1. For each $m \geq 1$, let $c_{m}$ be the vector in $l_{2}$ such that the first $m$ components of $c_{m}$ are 0 and the $n$-th component of $c_{m}$ is $2^{-n-1}$ for $n>m$; let $g_{m}$ be the function from $[0,1]$ to $l_{2}$ such that the $n$-th component of $g_{m}$ is $\left(1+w_{n-1}\right) / 2^{n}$ for $1 \leq n \leq m$ and the $n$-th component of $g_{m}$ is the constant $2^{-n-1}$ for $n>m$. Let $G_{m}(t)=\left\{c_{m}, g_{m}(t)\right\}$ for each $t \in[0,1]$. Let $g$ be the function $f$ in Example 1 and $c$ be the Bochner 
integral of $g$. Now let $Y$ be the space $\{0,1,1 / 2, \cdots, 1 / m, \cdots\}$ endowed with the usual metric. Let $G$ be the correspondence from $[0,1] \times Y$ to $l_{2}$ such that for each $t \in[0,1], G(t, 1 / m)=G_{m}(t)$ for each $m \geq 1$ and $G(t, 0)$ is the correspondence $F$ in Example 1. Then $G$ satisfies the conditions on norm (and hence weak) upper semicontinuity and measurability in Theorems 5 and 6 . Let $O=l_{2}-\{c / 2\}$. Then $O$ is an open neighborhood of $\int_{[0,1]} G(\cdot, 0) d \lambda$ in both norm and weak topologies. Since $c / 2 \in \int_{[0,1]} G(\cdot, 1 / m) d \lambda$ for each $m \geq 1, \int_{[0,1]} G(\cdot, 1 / m) d \lambda$ is not contained in $O$ for any $m \geq 1$. Therefore $\int_{[0,1]} G(\cdot, y) d \lambda$ is neither norm nor weakly upper semicontinuous at $y=0$.

Since bounded weak* closed sets are weak* compact, it is sometimes convenient to work with the weak* topology and the associated Gel'fand integral. In the rest of this section, we present some corresponding properties for the Gel'fand integral of a correspondence on a Loeb space. A function $f$ from a probability space $(T, \mathcal{T}, \nu)$ to the norm dual $X^{*}$ of a Banach space $X$ is said to be Gel'fand integrable if for each $x \in X, f(\cdot)(x)$ is integrable over $(T, \mathcal{T}, \nu)$. The Gel'fand integral of $f$ is the unique element $x^{*}$ in $X^{*}$ such that $x^{*}(x)=\int_{T} f(\cdot)(x) d \nu$ for all $x \in X$ (see [13, p. 53]), and $x^{*}$ is also denoted by $\int_{T} f d \nu$. Let $F$ be a correspondence from $(T, \mathcal{T}, \nu)$ to the dual $X^{*}$ and $\mathcal{W}_{F}$ be the collection of all Gel'fand integrable selections of $F$. Define the Gel'fand integral $\int_{T} F d \nu$ of the correspondence $F$ to be the set $\left\{\int_{T} f d \nu: f \in \mathcal{W}_{F}\right\}$ in $X^{*}$. Now we fix an arbitrarily given Loeb space $(\Omega, L(\mathcal{A}), L(P))$ and a general separable Banach space $X$.

Theorem 7. Let $F$ be a correspondence from $(\Omega, L(\mathcal{A}), L(P))$ to $X^{*}$. If the Loeb space $(\Omega, L(\mathcal{A}), L(P))$ is atomless, then the Gel'fand integral $\int_{\Omega} F d L(P)$ of $F$ is convex.

Theorem 8. Let $F$ be an integrably bounded, weak ${ }^{*}$ closed correspondence from a Loeb space $(\Omega, L(\mathcal{A}), L(P))$ to $X^{*}$. Then the Gel'fand integral $\int_{\Omega} F d L(P)$ is weak* compact.

Theorem 9. Let $F$ be an integrably bounded, weak* measurable, and weak ${ }^{*}$ closed valued correspondence from $(\Omega, L(\mathcal{A}), L(P))$ to $X^{*}$. If $(\Omega, L(\mathcal{A}), L(P))$ is atomless, then

$$
\int_{\Omega} w^{*}-\overline{c o} F d L(P)=\int_{\Omega} F d L(P)
$$

where $w^{*}-\overline{c o} F$ is the correspondence such that for each $\omega \in \Omega, w^{*}-\overline{c o} F(\omega)$ is the weak* closure of the convex hull of $F(\omega)$.

Theorem 10. Let $F$ be a weak $k^{*}$ closed valued correspondence from $\Omega \times Y$ to $X^{*}$, where $Y$ is a metric space. Assume that $F(\omega, y)$ is weak* measurable for each fixed $y$ and there is a real valued integrable function $g$ on $(\Omega, L(\mathcal{A}), L(P))$ such that for almost all $\omega \in \Omega, \sup \left\{\left\|x^{*}\right\|: x^{*} \in F(\omega, y)\right.$ for some $\left.y \in Y\right\} \leq g(\omega)$. Then if $F(\omega, y)$ is weak* upper semicontinuous on $Y$ for each fixed $\omega$, then $\int_{\Omega} F(\omega, y) d L(P)(\omega)$ is weak* $^{*}$ upper semicontinuous on $Y$; if $F(\omega, y)$ is weak ${ }^{*}$ lower semicontinuous on $Y$ for each fixed $\omega$, then $\int_{\Omega} F(\omega, y) d L(P)(\omega)$ is weak $k^{*}$ lower semicontinuous on $Y$; if $F(\omega, y)$ is weak ${ }^{*}$ continuous on $Y$ for each fixed $\omega$, then $\int_{\Omega} F(\omega, y) d L(P)(\omega)$ is weak* continuous on $Y$.

Remark. Let $F$ be the correspondence in Example 1. Then the Gel'fand integral of $F$ is the same as the Bochner integral of $F$. So the results in Theorems 7-9 fail on 
the unit Lebesgue interval. Similarly Theorem 10 also fails on the unit Lebesgue interval. The correspondence $G$ in Example 2 provides a counterexample for this case. Thus we see that all the ten theorems presented in this section fail on the unit Lebesgue interval.

\section{SOME PRELIMINARIES}

In this section we collect some results from [43] which are needed later. For a measurable mapping $g$ from a probability space $(T, \mathcal{T}, \nu)$ to a Polish space $X$, we use $\nu g^{-1}$ to denote the Borel probability measure on $X$ induced by $g$, which is often called the distribution of $g$. Let $\mathcal{M}(X)$ be the space of Borel probability measures on $X$ endowed with the topology of weak convergence of measures. For a correspondence $F$ from $(T, \mathcal{T}, \nu)$ to $X$, let

$$
\mathcal{D}_{F}=\left\{\nu f^{-1}: f \text { is a measurable selection of } F\right\} .
$$

We shall now fix a Loeb space $(\Omega, L(\mathcal{A}), L(P))$ and a Polish space $X$. The first proposition says that the set of distributions of the measurable selections of a correspondence on an atomless Loeb space is convex (see Theorem 2 in [43]).

Proposition 3.1. Let $F$ be a correspondence from $(\Omega, L(\mathcal{A}), L(P))$ to $X$. If $(\Omega, L(\mathcal{A}), L(P))$ is atomless, then $\mathcal{D}_{F}$ is a convex set in $\mathcal{M}(X)$.

The second proposition is about the compactness of the set of distributions of the measurable selections of a correspondence on a Loeb space (see Theorem 4 of $[43])$.

Proposition 3.2. Let $F$ be a compact valued correspondence from $(\Omega, L(\mathcal{A}), L(P))$ to $X$. Then $\mathcal{D}_{F}$ is compact in $\mathcal{M}(X)$. Moreover, if $F$ is measurable, then for any given $\varepsilon>0$, there is a compact set $K_{\varepsilon}$ in $X$ such that $L(P)\left(\left\{\omega: F(\omega) \subseteq K_{\varepsilon}\right\}\right)>$ $1-\varepsilon$.

In the following we present Kuratowski's notion of topological limits for a sequence of sets in a topological space (see [20, p. 15]).

Definition 3.3. Let $X$ be a topological space. If $A_{1}, A_{2}, \ldots$ are subsets of $X$, then by definition, $x \in \lim _{k \rightarrow \infty} \inf A_{k}$ if and only if every neighborhood of $x$ intersects all the $A_{k}$ with sufficiently large $k$; and $x \in \lim _{k \rightarrow \infty} \sup A_{k}$ if and only if every neighborhood of $x$ intersects infinitely many $A_{k}$. The limits are sometimes called topological limes inferior and superior respectively. If $\lim _{k \rightarrow \infty} \inf A_{k}=\lim _{k \rightarrow \infty} \sup A_{k}=A$, then we denote $\lim _{k \rightarrow \infty} A_{k}=A$.

The last result we collect here is used to prove results on the preservation of upper semicontinuity by integration (see Proposition 3.12 of [43]).

Proposition 3.4. Let $\left\{F_{n}\right\}_{n=1}^{\infty}$ be a sequence of measurable correspondences from $(\Omega, L(\mathcal{A}), L(P))$ to $X$ and let $F(\omega)=\lim _{n \rightarrow \infty} \sup F_{n}(\omega)$ for each $\omega \in \Omega$. Assume that there is a compact valued correspondence $G$ from $(\Omega, L(\mathcal{A}), L(P))$ to $X$ such that $L(P)\left\{\omega:\right.$ for any $\left.n \geq 1, F_{n}(\omega) \subseteq G(\omega)\right\}=1$. Then $\lim _{n \rightarrow \infty} \sup \mathcal{D}_{F_{n}} \subseteq \mathcal{D}_{F}$. 


\section{BOCHNER INTEGRATION OF CORRESPONDENCES}

Proof of Theorem 1. For any $y_{1}, y_{2} \in \int_{\Omega} F d L(P)$, there are Bochner integrable selections $f_{1}, f_{2}$ of $F$ such that $y_{i}=\int_{\Omega} f_{i} d L(P)$ for $i=1,2$. By Pettis's measurability theorem (see $\left[13\right.$, p. 42]), $f_{1}$ and $f_{2}$ are essentially norm separably valued. Thus we can assume that $f_{1}$ and $f_{2}$ take values from a separable Banach subspace $Y$ of $X$. Let $G$ be the correspondence from $\Omega$ to $Y$ such that $G(\omega)=\left\{f_{1}(\omega), f_{2}(\omega)\right\}$ for any $\omega \in \Omega$. By Proposition 3.1, the set $\mathcal{D}_{G}$ of distributions of the measurable selections of $G$ is convex. For $i=1,2$, let $\mu_{i}=L(P) g_{i}^{-1}$ be the probability measure on $Y$ induced by $g_{i}$. Then it can be checked that the identity mapping $I d_{Y}$ on $Y$ is Bochner integrable with respect to $\mu_{1}$ and $\mu_{2}$ and moreover $y_{i}=\int_{Y} I d_{Y} d \mu_{i}$ for $i=1,2$. It is clear that for any $\alpha \in[0,1]$, the Bochner integral $\int_{Y} I d_{Y} d\left(\alpha \mu_{1}+(1-\alpha) \mu_{2}\right)$ still exists and equals $\alpha y_{1}+(1-\alpha) y_{2}$. Since $\mathcal{D}_{G}$ is convex, there is a Bochner integrable selection $f$ of $G$ such that $L(P) f^{-1}=\alpha \mu_{1}+(1-\alpha) \mu_{2}$. Hence

$$
\alpha y_{1}+(1-\alpha) y_{2}=\int_{Y} I d_{Y} d L(P) f^{-1}=\int_{\Omega} f d L(P) \in \int_{\Omega} F d L(P),
$$

whence $\int_{\Omega} F d L(P)$ is convex.

Remarks. (1) As remarked earlier in Section 2, the convexity of the integrals of correspondences on atomless measure spaces implies the convexity of the ranges of those Banach space valued measures with Radon-Nikodým derivatives with respect to the atomless measure spaces. On the other hand, it is also known that results on the convexity of the ranges of vector measures imply the convexity of the integrals of correspondences on atomless measure spaces. In fact, as in the above proof, one can only work with a correspondence $F$ from an atomless measure space $(T, \mathcal{T}, \nu)$ to a Banach space $X$ by assuming $F(t)=\{f(t), g(t)\}$, where $f$ and $g$ are Bochner integrable functions. One may simply observe that

$$
\int_{T} F d \nu=\int_{T} g d \nu+\int_{T}\{0, f-g\} d \nu .
$$

Let $H$ be the vector measure defined by $H(A)=\int_{A}(f-g) d \nu$ for any $A \in \mathcal{T}$. If the range of $H$ is convex, then $\int_{T} F d \nu$ is convex.

(2) Next let $G$ be any $X$-valued measure on any given atomless Loeb space $(\Omega, L(\mathcal{A}), L(P))$ with a Radon-Nikodým derivative with respect to $L(P)$. Then by working with an internal lifting of $f$, one observes that $G$ is a vector Loeb measure as constructed in [33] and [42]. It is quite obvious that the range of a vector Loeb measure is closed (see Theorem 3.13 in [33]). The proof of Uhl's Lyapunov theorem clearly shows that the range of $G$ must be norm compact and convex (see Proposition 2.1 in Chapter IV of [41] or Proposition 4.5 in [42] for an earlier version of this observation). Thus Theorem 1 was already essentially shown in [41] and [42].

(3) For a correspondence $F$ from any given atomless Loeb space to a Banach space $X$, the Pettis integral of $F$ can be similarly defined. If $X$ is separable, then exactly the same proof as that of Theorem 1 shows that the Pettis integral of $F$ is still convex. Note that $F$ may have a measurable selection $f$ such that $\|f\|$ is not integrable. That is, the Pettis integral of $F$ is in general not the same as the Bochner integral of $F$.

(4) Let $\tau$ be a probability measure on the space $\mathcal{K}(X)$ of compact subsets of a separable Banach space $X$ with the Hausdorff metric. Let $H$ be the correspondence defined as the projection mapping from the product space of $(\mathcal{K}(X), \tau)$ with the 
unit Lebesgue interval $([0,1], \lambda)$ to $\mathcal{K}(X)$. It is known that the distribution of $H$ is $\tau$, and the set of distributions of measurable selections of $H$, which is convex and compact, realizes all possible distributions of selections of correspondences with distribution $\tau$ (see $[3,17]$ and $[18])$. Now let $(T, \mathcal{T}, \nu)$ be any atomless probability space. Then there is a measurable mapping $h$ from $T$ to $\mathcal{K}(X) \times[0,1]$ such that the distribution of $h$ is $\tau \otimes \lambda$. Let $G=H \circ h$. Then the distribution of $G$ is $\tau$, and $\int_{T} G d \nu$ is certainly convex and realizes all possible integrals of selections of correspondences with distribution $\tau$. Hence for a norm compact valued measurable correspondence $F$, there is a correspondence $G$ on any given atomless probability space (and of course including atomless Loeb spaces) such that $F$ and $G$ have the same distribution and $\int_{T} G d \nu$ is convex and realizes all possible integrals of selections of correspondences with the same distribution as $F$.

(5) After this paper was written up, the author received a preprint of D. Ross "A representation-independent integral for random closed sets" in which he considered the max-integral for a Radon random closed set (RRCS) taking values in a Banach space $X$. In that paper, the space $\mathcal{F}(X)$ of closed subsets of $X$ is endowed with Michael's topology, i.e., a subbasis is composed of sets of the form $\{A \in \mathcal{F}(X)$ : $A \cap O \neq \emptyset\})$ for an open set $O$ in $X$, and $\{A \in \mathcal{F}(X): A \cap E=\emptyset\})$ for a closed set $E$ in $X$. An RRCS is a measurable function from a probability space into $\mathcal{F}(X)$ with the Borel $\sigma$-algebra generated by Michael's topology such that its distribution on $\mathcal{F}(X)$ is Radon. For a Radon distribution $\sigma$ on $\mathcal{F}(X)$, the max-integral $\max (\sigma)$ is defined to be the set of all the integrals $\int_{X} x d \rho(x)$ for all Radon probability measures $\rho$ on $X$ satisfying $\rho(E) \leq \sigma(\{A \in \mathcal{F}(X): A \cap E \neq \emptyset\})$ for all closed subsets $E$ of $X$. It was noted there that there is one hyperfinite Loeb space with the counting measure $\left(\Omega_{\infty}, \mathcal{A}_{\infty}, P_{\infty}\right)$ and a RRCS $F_{\infty}$ in some $\beta$-saturated nonstandard model with $\beta>2^{\operatorname{card}(X)}$ such that the distribution of $F_{\infty}$ is $\sigma$, and $\int_{\Omega_{\infty}} F_{\infty} d P_{\infty}=\max (\sigma)$, which is convex. As remarked in (4), such a result is known for the case of normcompact valued measurable correspondences in the separable case. Next note that even if $X$ is separable, there is little information about Michael's topology on $\mathcal{F}(X)$. For example, it is not even known how the usual notion of closed valued measurable correspondences (see [4, 11], and [20]) is related to the notion of RRCSs. Also the larger saturation imposed on the nonstandard model was used there to work with topologies on $\mathcal{F}(X)$ and $X$, which excludes the natural hyperfinite Loeb counting spaces obtained from the ultrapower construction on a free ultrafilter on $\mathbb{N}$. Finally we note that we work with arbitrary atomless Loeb spaces in any $\aleph_{1}$-saturated nonstandard model.

Proof of Theorem 2. To show that $\int_{\Omega} F d L(P)$ is norm compact, it is enough to show that any sequence in $\int_{\Omega} F d L(P)$ has a subsequence norm convergent to some point in $\int_{\Omega} F d L(P)$. Pick a sequence $\left\{f_{n}\right\}_{n=1}^{\infty}$ of Bochner integrable selections of $F$. Let $x_{n}=\int_{\Omega} f_{n} d L(P)$ for each $n$. As above, by Pettis's measurability theorem, each $f_{n}$ is essentially norm separably valued. Thus we can assume that all the $f_{n}$ 's take values from a separable Banach subspace $Y$ of $X$. Let $G$ be a new correspondence from $\Omega$ to $Y$ such that for each $\omega \in \Omega, G(\omega)$ is the norm closure of the set $\left\{f_{n}(\omega): n \geq 1\right\}$ in $Y$. Then $G$ is an integrably bounded, norm compact valued, measurable correspondence from $(\Omega, L(\mathcal{A}), L(P))$ to the separable Banach space $Y$. Since $G$ is integrably bounded, there is a real valued integrable function $g$ on $(\Omega, L(\mathcal{A}), L(P))$ such that for almost all $\omega \in \Omega, \sup \{\|y\|: y \in G(\omega)\} \leq g(\omega)$. Thus for any given $\varepsilon>0$, there is a $\delta>0$ such that for any Loeb measurable set $A$ 
with $L(P)(A)<\delta$, we have $\int_{A} g d L(P)<\varepsilon / 2$. By Proposition 3.2, there is a norm compact subset $B_{\delta}$ in $Y$ such that

$$
L(P)\left\{\omega: G(\omega) \subseteq B_{\delta}\right\}>1-\delta .
$$

Let $K_{\delta}$ be the closed convex hull of $B_{\delta} \cup\{0\}$. Then Mazur's theorem (see [13, p. 51]) indicates that $K_{\delta}$ is still norm compact. Choose finitely many points $y_{1}, y_{2}, \ldots, y_{n}$ from $K_{\delta}$ such that for any $y \in K_{\delta}$, there is an integer $i$ with $1 \leq i \leq n$ such that $\left\|y-y_{i}\right\|<\varepsilon / 2$. Let $f$ be a measurable selection of $G$ and $A_{\delta}=f^{-1}\left(K_{\delta}\right)$. It is clear that $L(P)\left(\Omega-A_{\delta}\right)<\delta$. Thus

$$
\left\|\int_{A_{\delta}^{c}} f d L(P)\right\| \leq \int_{A_{\delta}^{c}} g d L(P)<\varepsilon / 2 .
$$

Note that $\int_{A_{\delta}} f d L(P)$ is still an element of $K_{\delta}$, which implies that there is a $y_{i}$ such that $\left\|\int_{A_{\delta}} f d L(P)-y_{i}\right\|<\varepsilon / 2$. Thus $\left\|\int_{\Omega} f d L(P)-y_{i}\right\|<\varepsilon$. Hence $\int_{\Omega} G d L(P)$ is totally bounded and thus relatively compact. Then the sequence $\left\{x_{n}\right\}_{n=1}^{\infty}$ has a norm convergent subsequence. Without loss of generality, we assume that $\lim _{n \rightarrow \infty} x_{n}=$ $x_{0}$. Let $\mu_{n}=L(P) f_{n}^{-1}$. Since $G$ is compact valued, Proposition 3.2 says that $\mathcal{D}_{G}$ is a compact subset of the space $\mathcal{M}(Y)$ of probability measures on $Y$. Without loss of generality, we assume that $\left\{\mu_{n}\right\}_{n=1}^{\infty}$ converges weakly to some $\mu$ in $\mathcal{D}_{G}$. The measure $\mu$ is induced by a measurable selection $f$ of $G$. It is easy to see that $f$ is a Bochner integrable selection of $F$. It remains to show that $x_{0}=\int_{\Omega} f d L(P)$. For any given continuous linear functional $\varphi$ on $Y$, it is clear that $\left\{\varphi \circ f_{n}\right\}_{n=1}^{\infty}$ converges to $\varphi \circ f$ in distribution. Since $F$ is integrably bounded, the sequence $\left\{\varphi \circ f_{n}\right\}_{n=1}^{\infty}$ is uniformly integrable. By Theorem 5.4 on p.32 of [9], we have

$$
\lim _{k \rightarrow \infty} \int_{\Omega} \varphi \circ f_{n} d L(P)=\int_{\Omega} \varphi \circ f d L(P) .
$$

Thus $\varphi\left(x_{0}\right)=\varphi\left(\int_{\Omega} f d L(P)\right)$, which implies that $x_{0}=\int_{\Omega} f d L(P)$. Therefore $\int_{\Omega} F d L(P)$ is norm compact.

Proof of Theorem 3. To show that $\int_{\Omega} F d L(P)$ is weakly compact, it is sufficient to show that it is weakly sequentially compact by the Eberlein-Smulian theorem (see [21, Section 18]). That is, any sequence in $\int_{\Omega} F d L(P)$ has a subsequence weakly convergent to some point in $\int_{\Omega} F d L(P)$. Pick a sequence $\left\{f_{n}\right\}_{n=1}^{\infty}$ of Bochner integrable selections of $F$. Let $x_{n}=\int_{\Omega} f_{n} d L(P)$ for each $n$. As in the proof of Theorem 2, we can assume that all the $f_{n}$ 's take values from a separable Banach subspace $Y$ of $X$. Let $G$ be a new correspondence from $\Omega$ to $Y$ such that for each $\omega \in \Omega, G(\omega)$ is the weak closure of the set $\left\{f_{n}(\omega): n \geq 1\right\}$ in $Y$. Since the space $Y$ is also weakly closed in $X, G(\omega)$ must be a weakly compact subset of $Y \cap F(\omega)$ for each $\omega \in \Omega$ by the assumption of weak compactness on $F$. Thus $G$ is a weakly compact valued, integrably bounded correspondence from $\Omega$ to the separable Banach space $Y$.

Next we show that for any bounded linear functional $\varphi$ on $Y, \varphi$ attains the maximum on the bounded set $\int_{\Omega} G d L(P)$. Without loss of generality, we assume that the norm of $\varphi$ is 1 . Let $a$ be the supremum value of $\varphi$ on $\int_{\Omega} G d L(P)$. Then there is a sequence $\left\{g_{n}\right\}_{n=1}^{\infty}$ of Bochner integrable selections of $G$ such that

$$
\lim _{n \rightarrow \infty} \varphi\left(\int_{\Omega} g_{n} d L(P)\right)=a .
$$


Since $Y$ is separable, the unit ball of the dual space $Y^{*}$ with the weak ${ }^{*}$ topology is separable (see Theorem 5.1 on p. 426 of [14]). Thus we can choose a countable dense set $\left\{\varphi_{n}\right\}_{n=1}^{\infty}$ in the unit ball of $Y^{*}$ with the weak* topology. We can take $\varphi_{1}=\varphi$. The metric

$$
\rho_{w}(x, y)=\sum_{n=1}^{\infty} \frac{1}{2^{n}}\left|\varphi_{n}(x-y)\right|
$$

defines a topology on $Y$ which is weaker than the weak topology on $Y$ (see also Theorem 6.3 on p. 434 of [14]). Thus the identity mapping $I d_{Y}$ on $Y$ is an injective continuous mapping from $Y$ with the norm topology to $Y$ with the topology induced by $\rho_{w}$. Let $\left(Z, \rho_{w}\right)$ be the completion of the metric space $\left(Y, \rho_{w}\right)$. Since $I d_{Y}$ is a Borel measurable injective mapping from the Polish space $Y$ with the norm topology to the Polish space $\left(Z, \rho_{w}\right)$, the Kuratowski theorem (see [34, p. 22]) says that $Y$ is a Borel subset of $\left(Z, \rho_{w}\right)$ and $I d_{Y}$ is a Borel isomorphism from the space $(Y,\|\cdot\|)$ to the space $\left(Y, \rho_{w}\right)$. Hence the Borel $\sigma$-algebras on $Y$ generated by the norm topology, the weak topology and the topology induced by $\rho_{w}$ are the same. Then $G$ is a compact valued measurable correspondence from $(\Omega, L(\mathcal{A}), L(P))$ to the Polish space $\left(Z, \rho_{w}\right)$. By Proposition 3.2, the set $\mathcal{D}_{G}$ of distributions of the measurable selections of $G$ is a compact subset of the space $\mathcal{M}(Z)$ of probability measures on $Z$. Then there is a measurable selection $g$ of $G$ and a subsequence $\left\{g_{n_{k}}\right\}_{k=1}^{\infty}$ such that the sequence $\left\{L(P) g_{n_{k}}^{-1}\right\}_{k=1}^{\infty}$ of measures on $\left(Z, \rho_{w}\right)$ converges weakly to the measure $L(P) g^{-1}$ on $\left(Z, \rho_{w}\right)$. It is easy to see that $g$ is a Bochner integrable selection of $G$. From the definition of $\rho_{w}$, we know that $\varphi$ is uniformly continuous on $\left(Y, \rho_{w}\right)$. Thus it has a unique continuous extension $\psi$ to $\left(Z, \rho_{w}\right)$. Hence $\left(L(P) g_{n_{k}}^{-1}\right) \psi^{-1}$ converges to $\left(L(P) g^{-1}\right) \psi^{-1}$ in distribution as $k \rightarrow \infty$. Note that not all bounded linear functionals on $Y$ are continuous on $\left(Y, \rho_{w}\right)$ except when $Y^{*}$ is norm separable (see [14, p. 426]). Since $\psi(g)=\varphi(g)$ and $\psi\left(g_{n}\right)=\varphi\left(g_{n}\right)$ for each $n \geq 1$, we know that $\left\{\varphi \circ g_{n_{k}}\right\}_{k=1}^{\infty}$ converges to $\varphi \circ g$ in distribution. Since $G$ is integrably bounded, the sequence $\left\{\varphi \circ g_{n_{k}}\right\}_{k=1}^{\infty}$ is uniformly integrable over $(\Omega, L(\mathcal{A}), L(P))$. As in the proof of Theorem 2, it follows from Theorem 5.4 on $\mathrm{p}$. 32 of $[9]$ that

$$
\lim _{k \rightarrow \infty} \int_{\Omega} \varphi \circ g_{n_{k}} d L(P)=\int_{\Omega} \varphi \circ g d L(P) .
$$

Thus we can obtain $a=\varphi\left(\int_{\Omega} g d L(P)\right)$, which says that $\varphi$ attains the maximum on $\int_{\Omega} G d L(P)$.

Now let $A$ be the weak closure of $\int_{\Omega} G d L(P)$ in $Y$. Then a maximum point of $\varphi$ on $\int_{\Omega} G d L(P)$ is still a maximum point of $\varphi$ on the bounded weakly closed set $A$. Hence a theorem of James (see [21, p. 157] or [23]) implies that $A$ is weakly compact and thus sequentially compact. Therefore the sequence $\left\{x_{n}\right\}_{n=1}^{\infty}$ in $\int_{\Omega} G d L(P)$ has a weakly convergent subsequence. Without loss of generality, assume that $\left\{x_{n}\right\}_{n=1}^{\infty}$ converges to some point $x_{0}$ in the weak topology of $Y$. Here we note that by a counterexample of James, a set $B$ in a Banach space is not necessarily weakly closed even if every bounded linear functional attains its maximum on $B$ (see [24]). To finish the proof of the theorem, we shall show that $x_{0} \in \int_{\Omega} F d L(P)$. Define the metric $\rho_{w}$ on $Y$ and obtain the space $Z$ as above. The same reason yields the fact that there is a Bochner integrable selection $f$ of $G$ and a subsequence $\left\{f_{n_{k}}\right\}_{k=1}^{\infty}$ such that $L(P) f_{n_{k}}^{-1}$ converges weakly to $L(P) f^{-1}$ on $\left(Z, \rho_{w}\right)$ as $k \rightarrow \infty$. By the procedure of the above paragraph and by the fact that $\varphi_{m}$ is uniformly continuous 
for any given $m \geq 1$, we can obtain

$$
\lim _{k \rightarrow \infty} \int_{\Omega} \varphi_{m} \circ f_{n_{k}} d L(P)=\int_{\Omega} \varphi_{m} \circ f d L(P),
$$

which implies that $\varphi_{m}\left(x_{0}\right)=\varphi_{m}\left(\int_{\Omega} f d L(P)\right)$. Since the sequence $\left\{\varphi_{m}\right\}_{m=1}^{\infty}$ separates points in $Y$, we have $x_{0}=\int_{\Omega} f d L(P)$ and hence $x_{0} \in \int_{\Omega} G d L(P)$, which clearly implies that $x_{0} \in \int_{\Omega} F d L(P)$. Therefore $\int_{\Omega} F d L(P)$ is weakly compact.

Proof of Theorem 4. By Theorem 8.6.2 on p. 327 of [4] (see also [19, p. 164] and $[25]$ ), we have

$$
\overline{\int_{\Omega} \overline{c o} F d L(P)}=\overline{c o} \int_{\Omega} F d L(P) .
$$

By the Krein-Smulian theorem (see [13, p. 51]), $\overline{c o} F$ is still a weakly compact valued correspondence. It is easy to see that $\overline{c o} F$ is also integrably bounded. By Theorem 3, both $\int_{\Omega} \overline{c o} F d L(P)$ and $\int_{\Omega} F d L(P)$ are weakly compact and hence norm closed. By Theorem 1, $\int_{\Omega} F d L(P)$ is convex. Therefore

$$
\int_{\Omega} \overline{c o} F d L(P)=\int_{\Omega} F d L(P),
$$

and we are done.

Remark. Let $(T, \mathcal{T}, \nu)$ be a positive measure space and $X$ a Banach space. Let $L_{\infty}(\nu)$ be the space of essentially bounded measurable functions on $(T, \mathcal{T}, \nu)$ and $L_{\infty}^{E}(\nu)$ the subspace of functions in $L_{\infty}(\nu)$ vanishing off $E$, where $\nu(E)>0$. For an $X$-valued vector measure $G$ which is absolutely continuous with respect to $\nu$, it was shown by Knowles in [30] that if for any $E \in \mathcal{T}$ with $\nu(E)>0$, the operator $\Phi_{E}: f \rightarrow \int_{E} f d G$ is not one-to-one on $L_{\infty}^{E}(\nu)$, then the range of $G$ is weakly compact and convex (see also p. 263 of the book [13] by Diestel and Uhl). Thus for a fixed Banach space $X$, if the measure space $(T, \mathcal{T}, \nu)$ is so large that for any $X$-valued measure absolutely continuous with respect to $\nu$, the operator $\Phi_{E}$ for each $E \in \mathcal{T}$ with $\nu(E)>0$ is always not one-to-one, then the range of $G$ is weakly compact and convex. That is certainly true, if the cardinality of $L_{\infty}^{E}(\nu)$ is greater than that of $X$ for all $E$ with $\nu(E)>0$. Since vector measures and correspondences are closely related to each other as remarked after the proof of Theorem 1, conditions of this sort should have implications for correspondences. In fact, it was suggested in [39] that if the algebraic dimension i.e., the cardinality of a Hamel basis of $L_{\infty}^{E}(\nu)$, is strictly greater than that of $X$ for all $E$ with $\nu(E)>0$, then $\int_{T} F d \nu=\int_{T} \overline{c o} F d \nu$ for a measurable weakly closed valued correspondence $F$ from $T$ into a weakly compact set $K$ in $X$. Note that this $F$ is not only weakly compact valued but also has a weakly compact set as a uniform upper bound. In order to work with a general separable Banach space, it is required in [39] (see p. 259) that the algebraic dimensions of $L_{\infty}^{E}(\nu)$ 's are strictly greater than the cardinality of the continuum for any $E \in \mathcal{T}$ with $\nu(E)>0$. However, we note that there is no natural measure space satisfying the required condition. It can be checked that the space of essentially bounded measurable functions with the $L_{\infty}$ norm on any hyperfinite Loeb space obtained from the ultrapower construction on a free ultrafilter on $\mathbb{N}$ has the cardinality of the continuum. Moreover, the space of essentially bounded measurable functions with the $L_{\infty}$-norm on the product space of $\aleph_{1}$ copies of the unit Lebesgue interval (the measure space suggested in Theorem 
4.1 of [39]) also has the cardinality of the continuum, and thus it does not satisfy the required condition either. From the point view of scientific modeling, when one needs to model a large finite phenomenon with no natural way of defining a distance on the relevant index set (such as names of certain social entities or sample points of experiments), one usually uses a measure space to model the index set. Since hyperfinite Loeb spaces already capture virtually all the asymptotic properties of the corresponding phenomenon being modeled, there is no need in scientific modeling to consider other types of measure spaces at all, and the technical advantages of hyperfinite Loeb spaces as shown in [43] and this paper highlight this point to a greater extent.

We are now ready to study sequences of correspondences. The first proposition concerns a property of the Bochner integral of the topological limit superior (see Definition 3.3) of a sequence of correspondences in the weak topology. The prefix $\mathrm{w}$ is added to refer the weak topology.

Proposition 4.1. Let $\left\{F_{n}\right\}_{n=1}^{\infty}$ be a sequence of measurable correspondences from $(\Omega, L(\mathcal{A}), L(P))$ to a separable Banach space $X$. Assume that there is an integrably bounded, weakly compact valued correspondence $G$ such that $F_{n}(\omega) \subseteq G(\omega)$ for each $n \geq 1$ and for each $\omega \in \Omega$. Let $F$ be the correspondence from $\Omega$ to $X$ such that $F(\omega)=w-\lim _{n \rightarrow \infty} \sup F_{n}(\omega)$ for each $\omega \in \Omega$. Then

$$
w-\lim _{n \rightarrow \infty} \sup \int_{\Omega} F_{n} d L(P) \subseteq \int_{\Omega} F d L(P) .
$$

Proof. It is sufficient to prove that for a sequence $\left\{f_{n}\right\}_{n=1}^{\infty}$ of Bochner integrable functions, if $f_{n}$ is a selection of $F_{n}$ for each $n \geq 1$ and w- $\lim _{n \rightarrow \infty} \int_{\Omega} f_{n} d L(P)=x_{0}$, then there is a Bochner integrable selection $f$ of $F$ such that $x_{0}=\int_{\Omega} f d L(P)$. As in the proof Theorem 3, we endow the separable Banach space $X$ with the metric $\rho_{w}$. The Polish space $\left(Z, \rho_{w}\right)$ is the completion of $\left(X, \rho_{w}\right)$. Then $G$ is a compact valued correspondence from $(\Omega, L(\mathcal{A}), L(P))$ to the Polish space $\left(Z, \rho_{w}\right)$. Since the Borel $\sigma$-algebras on $X$ generated by the norm topology and the topology by $\rho_{w}$ are the same, and since $X$ is a Borel subset of $\left(Z, \rho_{w}\right)$, the correspondences $F$ and $F_{n}$ are still measurable from $(\Omega, L(\mathcal{A}))$ to $\left(Z, \rho_{w}\right)$. It is also easy to see that the topology induced by $\rho_{w}$ on any weakly compact subset $B$ of $X$ is the same as the weak topology on $B$. Since for any $\omega \in \Omega$, the $F_{n}(\omega)$ 's are subsets of the weakly compact set $G(\omega)$, we know that the topological limit superior of the sequence $\left\{F_{n}(\omega)\right\}_{n=1}^{\infty}$ in $\left(Z, \rho_{w}\right)$ is still the same as w- $\lim _{n \rightarrow \infty} \sup F_{n}(\omega)$ in $X$ which is $F(\omega)$. By Proposition 3.4, we have $\lim _{n \rightarrow \infty} \sup \mathcal{D}_{F_{n}} \subseteq \mathcal{D}_{F}^{n \rightarrow \infty}$ in the space of probability measures on $\left(Z, \rho_{w}\right)$ with the topology of weak convergence of measures. Thus there is a subsequence $\left\{f_{n_{k}}\right\}_{k=1}^{\infty}$ and a measurable selection $f$ of $F$ such that $\left\{L(P) f_{n_{k}}^{-1}\right\}_{k=1}^{\infty}$ converges weakly to $L(P) f^{-1}$. The integrable boundedness of $G$ indicates that $f$ is Bochner integrable. As in the proof of Theorem 3, for each $m \geq 1, \varphi_{m}$ is uniformly continuous on $X$, where the $\varphi_{m}$ 's are the bounded linear functionals on $X$ which define the metric $\rho_{w}$. Then by the integrable boundedness of $G$ and by the procedure used in the proof of Theorems 2 and 3 , we can obtain

$$
\lim _{k \rightarrow \infty} \int_{\Omega} \varphi_{m} \circ f_{n_{k}} d L(P)=\int_{\Omega} \varphi_{m} \circ f d L(P),
$$

and hence $x_{0}=\int_{\Omega} f d L(P)$ and we are done. 
Remark. In the literature the type of results in Proposition 4.1 are usually referred to as Fatou's lemma for correspondences. For earlier versions, see [6, 7, 26, 40], and also see [46] for a survey. Note that if we take $F$ to be the correspondence in Example 1 in Section 2, then the constant sequence $\left\{F_{n}\right\}_{n=1}^{\infty}$ of correspondences with $F_{n}=F$ for each $n \geq 1$ provides a counterexample for Fatou's lemma in the setting of an infinite dimensional space, since

$$
\lim _{n \rightarrow \infty} \sup \int_{[0,1]} F_{n} d \lambda=\mathrm{w}-\lim _{n \rightarrow \infty} \sup \int_{[0,1]} F_{n} d \lambda=\overline{\int_{[0,1]} F d \lambda}
$$

which is not contained in $\int_{[0,1]} F d \lambda$ (see [38] for another counterexample).

The next proposition presents a property about the Bochner integral of the topological limit inferior of a sequence of correspondences on a Loeb space.

Proposition 4.2. Let $\left\{F_{n}\right\}_{n=1}^{\infty}$ be a sequence of measurable correspondences from $(\Omega, L(\mathcal{A}), L(P))$ to a separable Banach space $X$ and $F$ a correspondence satisfying $F(\omega)=w-\lim _{n \rightarrow \infty} \inf F_{n}(\omega)$ for each $\omega \in \Omega$. Assume that there is an integrably bounded, weakly compact valued correspondence $G$ such that $F_{n}(\omega) \subseteq G(\omega)$ for each $n \geq 1$ and for each $\omega \in \Omega$. Then

$$
\int_{\Omega} F d L(P) \subseteq w-\lim _{n \rightarrow \infty} \inf \int_{\Omega} F_{n} d L(P) .
$$

Proof. Let $x_{0}=\int_{\Omega} f d L(P)$ for some Bochner integrable selection $f$ of $F$. We have to show that there is a sequence $\left\{f_{n}\right\}_{n=1}^{\infty}$ of Bochner integrable functions such that $f_{n}$ is a selection of $F_{n}$ for each $n \geq 1$ and $\mathrm{w}-\lim _{n \rightarrow \infty} \int_{\Omega} f_{n} d L(P)=x_{0}$. As before, we endow the separable Banach space $X$ with the metric $\rho_{w}$ defined in the proof of Theorem 3. Let $\left(Z, \rho_{w}\right)$ be the completion of $\left(X, \rho_{w}\right)$. For each $\omega \in \Omega$, since the topology induced by $\rho_{w}$ on the weakly compact subset $G(\omega)$ of $X$ is the same as the weak topology on $G(\omega)$, we know that the topological limit inferior of the sequence $\left\{F_{n}(\omega)\right\}_{n=1}^{\infty}$ in $\left(Z, \rho_{w}\right)$ is still the same as w- $\lim _{n \rightarrow \infty} \inf F_{n}$, which is $F(\omega)$. Note that the correspondences $F$ and $F_{n}$ are still measurable from $(\Omega, L(\mathcal{A}))$ to $\left(Z, \rho_{w}\right)$. Thus, by Aumann's proof of his Proposition 5.1 in [6], we can find a measurable selection $f_{n}$ of $F_{n}$ for each $n \geq 1$ such that $\left\{f_{n}\right\}_{n=1}^{\infty}$ converges to $f$ everywhere with respect to the metric $\rho_{w}$. Thus for each $m \geq 1$, the continuity of $\varphi_{m}$ on $\left(X, \rho_{w}\right)$ yields the fact that $\left\{\varphi_{m} \circ f_{n}\right\}_{n=1}^{\infty}$ converges to $\varphi_{m} \circ f$ everywhere on $\Omega$, where the $\varphi_{m}$ 's are the bounded linear functionals which define the metric $\rho_{w}$ on $X$. The integrable boundedness of $G$ implies that

$$
\lim _{n \rightarrow \infty} \int_{\Omega} \varphi_{m} \circ f_{n} d L(P)=\int_{\Omega} \varphi_{m} \circ f d L(P)
$$

for each $m \geq 1$. Note that the $f_{n}$ 's are also Bochner integrable. Since for each $m \geq 1, \int_{\Omega} \varphi_{m} \circ f_{n} d L(P)=\varphi_{m}\left(\int_{\Omega} f_{n} d L(P)\right)$ and $\int_{\Omega} \varphi_{m} \circ f d L(P)=\varphi_{m}\left(\int_{\Omega} f d L(P)\right)$, we have $\lim _{n \rightarrow \infty} \rho_{w}\left(\int_{\Omega} f_{n} d L(P), \int_{\Omega} f d L(P)\right)=0$. It is clear that $\int_{\Omega} f d L(P)$ and $\int_{\Omega} f_{n} d L(P)$ for each $n \geq 1$ are elements of $\int_{\Omega} G d L(P)$, which is weakly compact by Theorem 3. Since the topology induced by $\rho_{w}$ agrees with the weak topology on the weakly compact set $\int_{\Omega} G d L(P)$, we have

$$
\text { w- } \lim _{n \rightarrow \infty} \int_{\Omega} f_{n} d L(P)=\int_{\Omega} f d L(P)
$$

and the proof is completed. 
Next we introduce Hausdorff semi-distances into our study of correspondences. Let $Y$ be a metric space with metric $d$. For a point $y \in Y$ and a nonempty subset $B$ of $Y$, let the distance $d(y, B)$ from the point $y$ to the set $B$ be $\inf _{z \in B} d(y, z)$. For nonempty subsets $A$ and $B$ of $Y$, define the Hausdorff semidistance $\sigma(A, B)=$ $\sup d(y, B)$. Note that the Hausdorff semidistance $\sigma$ is not symmetric. The Haus$y \in A$

dorff distance $\rho(A, B)$ between the sets $A$ and $B$ is defined to be the number $\max \{\sigma(A, B), \sigma(B, A)\}$ (see Chapter II of [11] and also [12]). Now we consider Hausdorff semi-distances induced by the norm of a separable Banach space $X$. Let $F, G$ be measurable correspondences and $f$ a measurable mapping from a probability space $(T, \mathcal{T}, \nu)$ to the Banach space $X$. By Lemma III.39 in [11], we know that $\sigma(F(t), G(t))$ and $d(f(t), G(t))$ are measurable functions on $(T, \mathcal{T})$. The result in Proposition 4.3 (1) below is essentially in Theorem 3.1 of [45]. However, our proof is is simpler than that of Theorem 3.1 in [45].

Proposition 4.3. Let $\left\{F_{n}\right\}_{n=0}^{\infty}$ be a sequence of measurable correspondences from a probability space $(T, \mathcal{T}, \nu)$ to a separable Banach space $X$. Assume that there is a real valued integrable function $g$ on $T$ such that for each $t \in T, \sup \{\|x\|: x \in$ $F_{n}(t)$ for some $\left.n \geq 0\right\} \leq g(t)$. Then

(1) if $\left\{\sigma\left(F_{0}(t), F_{n}(t)\right)\right\}_{n=1}^{\infty}$ converges to 0 in measure, then

$$
\lim _{n \rightarrow \infty} \sigma\left(\int_{T} F_{0} d \nu, \int_{T} F_{n} d \nu\right)=0
$$

(2) if $\left\{\sigma\left(F_{n}(t), F_{0}(t)\right)\right\}_{n=1}^{\infty}$ converges to 0 in measure, then

$$
\lim _{n \rightarrow \infty} \sigma\left(\int_{T} F_{n} d \nu, \int_{T} F_{0} d \nu\right)=0 .
$$

Proof. (1) It follows from the the definition of the Hausdorff semidistance that for each $n \geq 1$, there is a Bochner integrable selection $g_{n}$ of $F_{0}$ such that

$$
\sigma\left(\int_{T} F_{0} d \nu, \int_{T} F_{n} d \nu\right) \leq d\left(\int_{T} g_{n} d \nu, \int_{T} F_{n} d \nu\right)+\frac{1}{n} .
$$

For each $n \geq 1$ and for each $t \in T$, let $H_{n}(t)$ be the closed ball in $X$ with center $g_{n}(t)$ and radius $d\left(g_{n}(t), F_{n}(t)\right)+1 / n$; then $H_{n}(t) \cap F_{n}(t) \neq \emptyset$. It follows from Theorem III.41 in [11] that for each $n \geq 1, H_{n}$ is a measurable correspondence, and hence so is $H_{n} \cap F_{n}$. For each $n \geq 1$, let $f_{n}$ be a measurable selection of $H_{n} \cap F_{n}$; the integrable boundedness of $F_{n}$ implies that $f_{n}$ is Bochner integrable. Then for each $n \geq 1$ and each $t \in T$,

$$
\left\|g_{n}(t)-f_{n}(t)\right\| \leq d\left(g_{n}(t), F_{n}(t)\right)+\frac{1}{n} \leq \sigma\left(F_{0}(t), F_{n}(t)\right)+\frac{1}{n} .
$$

Hence for each $n \geq 1$,

$$
\begin{aligned}
\sigma\left(\int_{T} F_{0} d \nu, \int_{T} F_{n} d \nu\right) & \leq d\left(\int_{T} g_{n} d \nu, \int_{T} F_{n} d \nu\right)+\frac{1}{n} \leq\left\|\int_{T} g_{n} d \nu-\int_{T} f_{n} d \nu\right\|+\frac{1}{n} \\
& \leq \int_{T}\left\|g_{n}-f_{n}\right\| d \nu+\frac{1}{n} \leq \int_{T} \sigma\left(F_{0}, F_{n}\right) d \nu+\frac{2}{n} .
\end{aligned}
$$

Since $\sigma\left(F_{0}(t), F_{n}(t)\right) \leq 2 g(t)$ for all $t$ in $T$, the result follows from the scalar version of the dominated convergence theorem.

The proof for (2) is the same. 
The following corollary follows immediately from the above proposition.

Corollary 4.4. Let $\left\{F_{n}\right\}_{n=0}^{\infty}$ be a sequence of measurable correspondence from a probability space $(T, \mathcal{T}, \nu)$ to a separable Banach space $X$. Assume that the sequence $\left\{\rho\left(F_{n}(t), F_{0}(t)\right\}_{n=1}^{\infty}\right.$ converges to 0 in measure and there is an integrable function $g$ on $T$ such that for each $t \in T$,

$$
\sup \left\{\|x\|: x \in F_{n}(t) \text { for some } n \geq 0\right\} \leq g(t),
$$

where $\rho$ is the Hausdorff distance induced by the norm of $X$. Then

$$
\lim _{n \rightarrow \infty} \rho\left(\int_{T} F_{n} d \nu, \int_{T} F_{0} d \nu\right)=0 .
$$

Definition 4.5. Let $F$ be a correspondence from a topological space $X$ to a metric space $(Y, d)$. Let $\sigma$ be the Hausdorff semidistance induced by $d$ on the class of subsets of $Y$. Then $F$ is said to be Hausdorff upper (lower) semicontinuous at a point $x_{0}$ in $X$, if $\sigma\left(F(x), F\left(x_{0}\right)\right.$ ) (respectively, $\sigma\left(F\left(x_{0}\right), F(x)\right)$ ) is convergent to 0 as $x \rightarrow x_{0}$. If $F$ is both Hausdorff upper and lower semicontinuous at $x_{0}$, we say that $F$ is Hausdorff continuous at $x_{0}$.

The following is an immediate corollary of the above definition and Proposition 4.3. The Hausdorff semi-distance involved in the Banach space is defined from the norm.

Corollary 4.6. Let $(T, \mathcal{T}, \nu)$ be a probability space and $Y$ a metric space. Let $F$ be an integrably bounded correspondence from $T \times Y$ to a separable Banach space $X$. Assume that $F(t, y)$ is measurable for each fixed $y \in Y$. Then if $F(t, y)$ is Hausdorff upper semicontinuous on $Y$ for each fixed $t \in T$, then $\int_{T} F(t, y) d \nu(t)$ is Hausdorff upper semicontinuous on $Y$; if $F(t, y)$ is Hausdorff lower semicontinuous on $Y$ for each fixed $t$, then $\int_{T} F(t, y) d \nu(t)$ is Hausdorff lower semicontinuous on $Y$; if $F(t, y)$ is Hausdorff continuous on $Y$ for each fixed $t$, then $\int_{T} F(t, y) d \nu(t)$ is Hausdorff continuous on $Y$.

Remark. In [45], a notion of quasi upper semicontinuity is introduced, which is the same as our Hausdorff upper semicontinuity.

In the following proposition we present a property about the Bochner integral of the topological limit superior of a sequence of correspondences in the norm topology.

Proposition 4.7. Let $\left\{F_{n}\right\}_{n=1}^{\infty}$ be a sequence of measurable correspondences from $(\Omega, L(\mathcal{A}), L(P))$ to a separable Banach space $X$. Assume that there is an integrably bounded norm compact valued correspondence $G$ such that $F_{n}(\omega) \subseteq G(\omega)$ for each $\omega$ in $\Omega$ and each $n \geq 1$. Let $F=\lim _{n \rightarrow \infty} \sup F_{n}$. Then

$$
\lim _{n \rightarrow \infty} \sup \int_{\Omega} F_{n} d L(P) \subseteq \int_{\Omega} F d L(P) .
$$

Proof. First we note that for a sequence $\left\{A_{n}\right\}_{n=0}^{\infty}$ of sets in a compact metric space with $A_{0}$ compact, $\lim _{n \rightarrow \infty} \sup A_{n} \subseteq A_{0}$ if and only if the Hausdorff semi-distance $\sigma\left(A_{n}, A_{0}\right)=0$ goes to 0 as $n \rightarrow \infty$ (see the proof of Theorem 1 on p. 17 of $[20])$. Since for each $\omega \in \Omega, F(\omega)$ is a closed subset of the norm compact set $G(\omega), F(\omega)$ is norm compact. Thus the above fact implies that for each $\omega \in \Omega$, 
$\lim _{n \rightarrow \infty} \sigma\left(F_{n}(\omega), F_{0}(\omega)\right)=0$. Hence by Proposition 4.3, we have

$$
\lim _{n \rightarrow \infty} \sigma\left(\int_{\Omega} F_{n} d \nu, \int_{\Omega} F_{0} d \nu\right)=0 .
$$

By Theorem 2, we know that $\int_{\Omega} F d L(P)$ is norm compact. By another appeal to the above fact, we have

$$
\lim _{n \rightarrow \infty} \sup \int_{\Omega} F_{n} d L(P) \subseteq \int_{\Omega} F_{0} d L(P),
$$

and we are done.

The following proposition is an extension of Aumann's Proposition 5.1 in [6] to the setting of Banach space valued correspondences.

Proposition 4.8. Let $\left\{F_{n}\right\}_{n=1}^{\infty}$ be a sequence of measurable correspondences from $(T, \mathcal{T}, \nu)$ to a separable Banach space $X$ and let $F$ be a correspondence defined by $F(t)=\lim _{n \rightarrow \infty} \inf F_{n}(t)$ for each $t \in T$. Assume that there is a real valued integrable function $g$ on $T$ such that for each $t \in T, \sup \left\{\|x\|: x \in F_{n}(t)\right.$ for some $\left.n \geq 1\right\} \leq$ $g(t)$. Then

$$
\int_{T} F d \nu \subseteq \lim _{n \rightarrow \infty} \inf \int_{T} F_{n} d \nu
$$

Proof. Let $x_{0}=\int_{T} f d \nu$ for some Bochner integrable selection $f$ of $F$. By using the idea in the proof of Proposition 5.1 in [6], we can find a sequence $\left\{f_{n}\right\}_{n=1}^{\infty}$ of measurable functions such that $f_{n}$ is a selection of $F_{n}$ and $\left\{f_{n}\right\}_{n=1}^{\infty}$ converges in norm to $f$ everywhere in $T$. Then by the Dominated Convergence Theorem for Bochner integrals (see [13, p. 45]), we have $\lim _{n \rightarrow \infty} \int_{T} f_{n} d \nu=x_{0}$. The result follows.

By the results we have proven, we can obtain the following two versions of the dominated convergence theorem for the Bochner integral of Banach space valued correspondences (see also [45]).

Corollary 4.9 (Strong Dominated Convergence Theorem). Let $\left\{F_{n}\right\}_{n=1}^{\infty}$ be a sequence of measurable correspondence from a Loeb space $(\Omega, L(\mathcal{A}), L(P))$ to a separable Banach space $X$. Assume that there is an integrably bounded, norm compact valued correspondence $G$ such that for each $n \geq 1, F_{n}(\omega) \subseteq G(\omega)$ for almost all $\omega$ in $\Omega$. If $\lim _{n \rightarrow \infty} F_{n}=F$, then

$$
\lim _{n \rightarrow \infty} \int_{\Omega} F_{n} d L(P)=\int_{\Omega} F d L(P) .
$$

Proof. By Propositions 4.7 and 4.8, we have

$$
\lim _{n \rightarrow \infty} \sup \int_{\Omega} F_{n} d L(P) \subseteq \int_{\Omega} F d L(P) \subseteq \lim _{n \rightarrow \infty} \inf \int_{\Omega} F_{n} d L(P),
$$

which implies the result.

Corollary 4.10 (Weak Dominated Convergence Theorem). Let $\left\{F_{n}\right\}_{n=1}^{\infty}$ be a sequence of measurable correspondences from $(\Omega, L(\mathcal{A}), L(P))$ to a separable Banach 
space $X$. Assume that there is an integrably bounded, weakly compact valued correspondence $G$ such that for every $n, F_{n}(\omega) \subseteq G(\omega)$ for almost all $\omega$ in $\Omega$. If $w-\lim _{n \rightarrow \infty} F_{n}=F$, then

$$
w-\lim _{n \rightarrow \infty} \int_{\Omega} F_{n} d L(P)=\int_{\Omega} F d L(P) .
$$

Proof. By Propositions 4.1 and 4.2, we have

$$
\mathrm{w}-\lim _{n \rightarrow \infty} \sup \int_{\Omega} F_{n} d L(P) \subseteq \int_{\Omega} F d L(P) \subseteq \mathrm{w}-\lim _{n \rightarrow \infty} \inf \int_{\Omega} F_{n} d L(P) .
$$

Then the result follows immediately.

Proof of Theorem 5. Since $G$ is norm compact valued, Theorem 2 implies that $\int_{\Omega} G d L(P)$ is norm compact. By the assumption that $G$ dominates $F(\cdot, y)$ for each $y \in Y$, we know that $\int_{\Omega} F(\cdot, y) d L(P)$ induces a closed valued correspondence from $Y$ to the compact space $\int_{\Omega} G d L(P)$. Note that a closed valued correspondence from a metrizable space to a compact metrizable space is upper semicontinuous if and only if it has a closed graph (see Proposition 1.4 .8 on p. 42 of [4]). Let $\left\{x_{n}\right\}_{n=0}^{\infty}$ be a sequence in $X$ and $\left\{y_{n}\right\}_{n=0}^{\infty}$ be a sequence in $Y$ such that for each $n \geq 1$, $x_{n} \in \int_{\Omega} F\left(\cdot, y_{n}\right) d L(P), \lim _{n \rightarrow \infty} x_{n}=x_{0}$, and $\lim _{n \rightarrow \infty} y_{n}=y_{0}$. We have to show that $x_{0} \in \int_{\Omega} F\left(\cdot, y_{0}\right) d L(P)$. It is clear that $x_{0} \in \lim _{n \rightarrow \infty} \sup \int_{\Omega} F\left(\cdot, y_{n}\right) d L(P)$. Proposition 4.7 yields the fact that $x_{0} \in \int_{\Omega} \lim _{n \rightarrow \infty} \sup F\left(\cdot, y_{n}\right) d L(P)$. For each $\omega \in \Omega$, since the correspondence $F(\omega, \cdot)$ has a closed graph, we have $\lim _{n \rightarrow \infty} \sup F\left(\omega, y_{n}\right) \subseteq F\left(\omega, y_{0}\right)$. Hence $x_{0} \in \int_{\Omega} F\left(\cdot, y_{0}\right) d L(P)$, and thus the result on the preservation of norm upper semicontinuity by integration is shown.

For lower semicontinuity, we use a characterization of lower semicontinuity by sequences (see [20, p. 27]). Let $\left\{y_{n}\right\}_{n=0}^{\infty}$ be a sequence in $Y$ with $\lim _{n \rightarrow \infty} y_{n}=y_{0}$. For each $\omega \in \Omega$, since the correspondence $F(\omega, \cdot)$ is assumed to be lower semicontinuous, we have $F\left(\omega, y_{0}\right) \subseteq \lim _{n \rightarrow \infty} \inf F\left(\omega, y_{n}\right)$. Hence Proposition 4.8 allows us to claim that $\int_{\Omega} F\left(\cdot, y_{0}\right) d L(P) \subseteq \lim _{n \rightarrow \infty} \inf \int_{\Omega} F\left(\omega, y_{n}\right) d L(P)$, which implies the desired result.

Proof of Theorem 6. As in the proof of Theorem 3, we note that the weak topology on a weakly compact set in a separable Banach space is metrizable. Then by using the same idea as in the proof of Theorem 5, we can obtain the results on the preservation of weak upper and lower semicontinuity by integration from Propositions 4.1 and 4.2 .

Remark. Let $K$ be a fixed weakly compact and convex set in a separable Banach space $X$. As noted in the proof of Theorem 3 and Proposition $4.1, K$ is also metrizable. It can be checked that the mapping $L$ from the space of Borel probability measures $\mathcal{M}(K)$ to $K$ defined by $L(\nu)=\int_{x \in K} x d \nu(x)$ is continuous, where $\mathcal{M}(K)$ is endowed with the topology of weak convergence of measures and $K$ with the subspace topology induced by the weak topology on $X$. Then for a correspondence $G$ from a Loeb space $(\Omega, L(\mathcal{A}), L(P))$ to $K$, it is easy to see that the integral $\int_{\Omega} G d L(P)$ is the set $L\left(\mathcal{D}_{G}\right)$. By using this property together with the fact that $L$ is linear and continuous, it is easy to obtain from the propositions in Section 3 the corresponding convexity, weak compactness and preservation of weak upper semicontinuity for integrals. When $K$ is norm compact and convex, the same type 
of results for the norm topology can be derived in a similar way. Moreover, when $K$ is compact, convex, and metrizable in some topological vector space, if one uses a suitable notion of integral so that the defined linear operator $L$ is still continuous (this should be the usual case), then one can also obtain the same results immediately. For example, for the Gel'fand integral to be discussed in the next section, if one restricts the considered correspondences to be bounded, then the corresponding results follow from the propositions in Section 3 easily. Since our Theorems 1-10 allow correspondences not dominated by any fixed compact set, such a method will not work for this general case.

As claimed earlier, once we have results on a hyperfinite Loeb space, then the usual techniques of lifting, pushing-down, and transfer can be used to obtain asymptotic results for the large finite case (see [1, 2, 31], and [36]). As an example, we consider here a simple case by obtaining a result on the approximate convexity of the weighted average of a large finite number of sets in a Banach space.

For each $n \geq 1$, let $\left\{c_{i}^{n}\right\}_{i=1}^{n}$ be a finite sequence of nonnegative real numbers with sum 1 and $\left\{K_{i}^{n}\right\}_{i=1}^{n}$ a finite sequence of nonempty sets in $X$. Let $\left(\Omega_{n}, \mathcal{A}_{n}, P_{n}\right)$ be a finite probability space, where $\Omega_{n}=\{1,2, \ldots, n\}, \mathcal{A}_{n}=\mathcal{P}\left(\Omega_{n}\right)$ the power set of $\Omega_{n}$, and $P_{n}(A)=\sum_{i \in A} c_{i}^{n}$ for each $A \subseteq \Omega_{n}$. Define a correspondence $F_{n}$ on $\Omega_{n}$ by letting $F_{n}(i)=K_{i}^{n}$ for each $i \in \Omega_{n}$. Then the set

$$
A_{n}=\int_{\Omega_{n}} F_{n} d P_{n}=\sum_{i=1}^{n} c_{i}^{n} K_{i}^{n}
$$

is a weighted average of the sets $K_{i}^{n}$. Define a function $\varphi_{n}$ on $\Omega_{n}$ by letting $\varphi_{n}(i)$ be the supremum norm of the vectors in the set $K_{i}^{n}$. Assume that the following type of uniform integrability condition on the $\varphi_{n}$ 's holds:

$$
\lim _{m \rightarrow \infty} \sup _{1 \leq n<\infty} \int_{\varphi_{n}>m} \varphi_{n} d P_{n}=0 .
$$

Note that if the sets $K_{i}^{n}$ are uniformly bounded, then the uniform integrability condition is certainly satisfied.

Proposition 4.11. Assume that $\lim _{n \rightarrow \infty 1 \leq i \leq n} \max _{i}=0$ and also the following tightness condition is satisfied: for any $\varepsilon>0$, there is a norm compact set $K_{\varepsilon}$ in $X$ such that for any $n \geq 1$,

$$
P_{n}\left(\left\{i: K_{i}^{n} \subseteq K_{\varepsilon}\right\}\right)>1-\varepsilon .
$$

Then for any given $\varepsilon>0$, there is a positive integer $N$ such that for any $n>N$, the following holds: for any $\alpha \in[0,1]$ and for any $x_{1}, x_{2} \in A_{n}$, there is a point $y \in A_{n}$ such that $\left\|y-\left(\alpha x_{1}+(1-\alpha) x_{2}\right)\right\|<\varepsilon$.

Proof. Transfer the sequences of $K_{i}^{n}$ 's, $c_{i}^{n}$ 's to obtain $\left\{{ }^{*} c_{i}^{n}: n \in{ }^{*} \mathbb{N}, 1 \leq i \leq n\right\}$ and $\left\{{ }^{*} K_{i}^{n}: n \in{ }^{*} \mathbb{N}, 1 \leq i \leq n\right\}$. Define the internal sequences $\left\{\left(\Omega_{n}, \mathcal{A}_{n}, P_{n}\right)\right\}_{n \in * \mathbb{N}}$ and $\left\{F_{n}\right\}_{n \in * \mathbb{N}}$ accordingly. For any given $\eta \in{ }^{*} \mathbb{N}_{\infty}$, let $\left(\Omega_{\eta}, L\left(\mathcal{A}_{\eta}\right), L\left(P_{\eta}\right)\right)$ be the corresponding Loeb space of the internal probability space $\left(\Omega_{\eta}, \mathcal{A}_{\eta}, P_{\eta}\right)$. The conditions on the $c_{i}^{n}$ 's implies that this Loeb space is atomless. The tightness condition implies that for $L\left(P_{\eta}\right)$-almost all $i \in \Omega_{\eta}, F_{\eta}(i)$ is contained in the star transform of some norm compact set in $X$, and so we can define a correspondence $G_{\eta}$ from $\Omega_{\eta}$ to $X$ such that for $L\left(P_{\eta}\right)$-almost all $i \in \Omega_{\eta}, G_{\eta}(i)=\left\{{ }^{\circ} x: x \in F_{\eta}(i)\right\}$, 
where ${ }^{\circ} x$ is the standard part of $x$ with respect to the norm topology of $X$. It follows from Theorem 1 that $\int_{\Omega_{\eta}} G_{\eta} d L\left(P_{\eta}\right)$ is convex.

The uniform integrability of the $\varphi_{n}$ 's implies that for any $m \in{ }^{*} \mathbb{N}_{\infty}$,

$$
\sup _{n \in \mathbb{N}} \int_{\varphi_{n}>m} \varphi_{n} d P_{n} \simeq 0
$$

By Proposition 2.35 on p. 187 of [22], we know that each $\varphi_{\eta}$ is $S$-integrable. That is,

$$
\int_{\Omega_{\eta}}{ }^{\circ} \varphi_{\eta} d L\left(P_{\eta}\right)={ }^{\circ} \int_{\Omega_{\eta}} \varphi_{\eta} d P_{\eta}<\infty .
$$

Hence the correspondence $G_{\eta}$ is integrably bounded.

Now for any $x_{1}, x_{2} \in A_{\eta}$, there are internal selections $f_{1}, f_{2}$ of $F_{\eta}$ such that for $j=1,2, \int_{\Omega_{\eta}} f_{j} d P_{\eta}=x_{j}$. It is clear that ${ }^{\circ} f_{1}$ and ${ }^{\circ} f_{2}$ are Bochner integrable selections of $G_{\eta}$. One can check that $\int_{\Omega_{\eta}}{ }^{\circ} f_{j} d L\left(P_{\eta}\right) \simeq x_{j}$ for $j=1,2$. For any $\alpha \in{ }^{*}[0,1]$, the convexity of $\int_{\Omega_{\eta}} G_{\eta} d L\left(P_{\eta}\right)$ implies that there is a Bochner integrable selection $g$ of $G_{\eta}$ such that

$$
\int_{\Omega_{\eta}} g d L\left(P_{\eta}\right)={ }^{\circ} \alpha \int_{\Omega_{\eta}}{ }^{\circ} f_{1} d L\left(P_{\eta}\right)+\left(1-{ }^{\circ} \alpha\right) \int_{\Omega_{\eta}}{ }^{\circ} f_{2} d L\left(P_{\eta}\right) .
$$

By the usual lifting procedure, there is an internal selection $h$ of $F_{\eta}$ such that for $L\left(P_{\eta}\right)$-almost all $i \in \Omega_{\eta},{ }^{\circ} h(i)=g(i)$ and $\int_{\Omega_{\eta}} h d P_{\eta} \simeq \int_{\Omega_{\eta}} g d L\left(P_{\eta}\right)$. Thus $\int_{\Omega_{\eta}} h d P_{\eta} \simeq \alpha x_{1}+(1-\alpha) x_{2}$. Let $y=\int_{\Omega_{\eta}} h d P_{\eta}$. Then $y \in A_{\eta}$. Hence, for any $\varepsilon \in \mathbb{R}^{+}$, the following is true for all $\eta \in{ }^{*} \mathbb{N}_{\infty}$ :

$$
\left(\forall x_{1} \in A_{\eta}\right)\left(\forall x_{2} \in A_{\eta}\right)\left(\forall \alpha \in{ }^{*}[0,1]\right)\left(\exists y \in A_{\eta}\right)\left(\left\|y-\left(\alpha x_{1}+(1-\alpha) x_{2}\right)\right\|<\varepsilon\right)
$$

Thus the result follows from the Permanence Principle (see [22, p. 100]).

Remarks. (1) If we have asymptotic results for the large finite case, then by transfer and pushing-down, we can obtain exact results on a hyperfinite Loeb space; but we may not be able to obtain corresponding exact results on the unit Lebesgue interval.

(2) If the norm tightness is replaced by weak tightness, i.e., the sets $K_{\varepsilon}$ in Proposition 4.11 are only required to be weakly compact in a separable Banach space, then we can use the metric $\rho_{w}$ as in the proof of Theorem 3 to replace $\left\|y-\left(\alpha x_{1}+(1-\alpha) x_{2}\right)\right\|<\varepsilon$ by $\rho_{w}\left(y,\left(\alpha x_{1}+(1-\alpha) x_{2}\right)\right)<\varepsilon$ in the statement of Proposition 4.11, and obtain a similar result on approximate convexity. The proof will be identical to the above except at those places involving the norm, where the metric $\rho_{w}$ will be used. Note that the topology on the $K_{\varepsilon}$ 's induced by $\rho_{w}$ is the same as the weak topology.

\section{Gel'FAnd integration of CORRESPONDENCES}

In the final section, we shall consider Gel'fand integral in the norm dual of a separable Banach space. Hereafter all integrals are Gel'fand integrals unless otherwise noted. We shall fix a separable Banach space $X$ and a Loeb probability space $(\Omega, L(\mathcal{A}), L(P))$. Let $X^{*}$ be the norm dual of $X$. Since $X$ is separable, we can choose a sequence $\left\{x_{m}\right\}_{m=1}^{\infty}$ from the unit ball of $X$ such that the linear space 
spanned by the sequence is dense in $X$. Define a metric $d_{w}$ on the dual $X^{*}$ by letting

$$
d_{w}\left(x^{*}, y^{*}\right)=\sum_{m=1}^{\infty} \frac{1}{2^{m}}\left|x^{*}\left(x_{m}\right)-y^{*}\left(x_{m}\right)\right|
$$

for each pair of $x^{*}, y^{*}$ in $X^{*}$ (see [14, p. 426]). It is easy to verify that this topology is weaker than the weak ${ }^{*}$ topology on $X^{*}$. Thus the two topologies agree on any weak* compact subset of $X^{*}$ and hence on any norm bounded set in $X^{*}$ by the Alaoglu theorem. Let $\left(Z, d_{w}\right)$ be the completion of the metric space $\left(X^{*}, d_{w}\right)$. Since $X^{*}$ is a union of countably many weak* compact sets, we know that $X^{*}$ is a $\sigma$ compact set and hence a Borel set in $\left(Z, d_{w}\right)$. It is clear that the Borel $\sigma$-algebras on $X^{*}$ generated by the weak* topology and the topology induced by the metric $d_{w}$ are the same. Thus weak ${ }^{*}$ measurability and measurability with respect to $\left(X^{*}, d_{w}\right)$ are the same. We shall also use measurable to refer weak* measurable in the rest of the section. For some earlier work on Gel'fand integrals on Loeb spaces, see [42]. In [25], a version of the result for Gel'fand integrals in the form of Theorem 8.6.2 on p. 327 of [4] (see also [19, p. 164] and Theorem 1 of [25]) is given. Note that the arguments used in this section are similar to those used in Section 4.

Proof of Theorem 7. We can obtain a proof of this theorem by some minor changes in the proof of Theorem 1, which is left for the readers to check.

Remark. As in Proposition 4.11, we can obtain approximate convexity for sets in the setting of weak* topologies. We simply use the metric $d_{w}$ defined above to replace $\left\|y-\left(\alpha x_{1}+(1-\alpha) x_{2}\right)\right\|<\varepsilon$ by $d_{w}\left(y,\left(\alpha x_{1}+(1-\alpha) x_{2}\right)\right)<\varepsilon$ in the statement of Proposition 4.11, and obtain a similar result on approximate convexity. The proof is again identical to that of Proposition 4.11 except at those places involving the norm, where the metric $d_{w}$ will be used. Note that in this case no additional tightness condition is needed, since the uniform integrability condition guarantees weak* tightness.

Proof of Theorem 8. Since $F$ is integrably bounded, the integral $\int_{\Omega} F d L(P)$ of $F$ is a bounded set in $X^{*}$. Thus we only have to show that $\int_{\Omega} F d L(P)$ is closed in $\left(X^{*}, d_{w}\right)$. Let $\left\{y_{n}\right\}_{n=1}^{\infty}$ be a sequence in $\int_{\Omega} F d L(P)$ such that $\lim _{n \rightarrow \infty} d_{w}\left(y_{n}, y\right)=0$ for some $y \in X^{*}$. There is a sequence $\left\{f_{n}\right\}_{n=1}^{\infty}$ of Gel'fand integrable selections of $F$ such that $y_{n}=\int_{\Omega} f_{n} d L(P)$ for each $n \geq 1$. It is clear that $F$ is a compact valued correspondence from the Loeb space $(\Omega, L(\mathcal{A}), L(P))$ to the Polish space $\left(Z, d_{w}\right)$. By Proposition 3.2, the set $\mathcal{D}_{F}$ of distributions of the measurable selections of $F$ is compact. Thus the sequence $\left\{L(P) f_{n}^{-1}\right\}_{n=1}^{\infty}$ of measures on $Z$ has a weakly convergent subsequence with limit in $\mathcal{D}_{F}$. Without loss of generality, we assume $\left\{L(P) f_{n}^{-1}\right\}_{n=1}^{\infty}$ converges weakly to $L(P) f^{-1}$ for some measurable selection $f$ of $F$. It follows from the definition of $d_{w}$ that for each $m \geq 1, x_{m}$ induces a uniformly continuous function $x_{m}^{* *}$ on $X^{*}$. The arguments used in the proof of Theorem 3 indicate that $\left\{x_{m}^{* *} \circ f_{n}\right\}_{n=1}^{\infty}$ converges to $x_{m}^{* *} \circ f$ in distribution. Since $F$ is integrably bounded, we know that $f$ is Gel'fand integrable and the sequence $\left\{x_{m}^{* *} \circ f_{n}\right\}_{n=1}^{\infty}$ is uniformly integrable over $(\Omega, L(\mathcal{A}), L(P))$ for each $m \geq 1$. As before, Theorem 5.4 on p. 32 of [9] yields the fact that

$$
\lim _{n \rightarrow \infty} \int_{\Omega} x_{m}^{* *} \circ f_{n} d L(P)=\int_{\Omega} x_{m}^{* *} \circ f d L(P)
$$


for each $m \geq 1$, which implies that $\lim _{n \rightarrow \infty} d_{w}\left(y_{n}, \int_{\Omega} f d L(P)\right)=0$. Hence $y \in$ $\int_{\Omega} F d L(P)$. Thus $\int_{\Omega} F d L(P)$ is closed in $\left(X^{*}, d_{w}\right)$, and the proof is complete.

Proof of Theorem 9. Suppose that there is a Gel'fand integrable selection $f$ of $\mathrm{w}^{*}-\overline{c o} F$ such that $y_{0}=\int_{\Omega} f d L(P) \notin \int_{\Omega} F d L(P)$. By Theorem $8, \int_{\Omega} F d L(P)$ is compact in $\left(X^{*}, d_{w}\right)$. Thus the distance $d_{w}\left(y_{0}, \int_{\Omega} F d L(P)\right)$ between $y_{0}$ and $\int_{\Omega} F d L(P)$ is positive. Since $F$ is integrably bounded, there is an integrable real valued function $g$ on $(\Omega, L(\mathcal{A}), L(P))$ such that for each $\omega \in \Omega, \sup \left\{\left\|x^{*}\right\|: x^{*} \in\right.$ $F(\omega)\} \leq g(\omega)$. Pick $M>\int_{\Omega} g d L(P)$. We can choose $n$ large enough such that $d_{w}\left(y_{0}, \int_{\Omega} F d L(P)\right)>3 M / 2^{n}$. Thus for any $y \in \int_{\Omega} F d L(P)$,

$$
\sum_{m=1}^{n} \frac{1}{2^{m}}\left|x_{m}^{* *}\left(y_{0}-y\right)\right|>\frac{3 M}{2^{n}}-\sum_{m=n+1}^{\infty} \frac{2 M}{2^{n}}=\frac{M}{2^{n}} .
$$

Let $h$ be the mapping from $X^{*}$ to $\mathbb{R}^{n}$ such that $h(y)=\left(x_{1}^{* *}(y), \ldots, x_{n}^{* *}(y)\right)$ for any $y \in X^{*}$. Then it is clear that $h\left(y_{0}\right)$ is not in the compact set $h\left(\int_{\Omega} F d L(P)\right)$ in $\mathbb{R}^{n}$. Let $h \circ F$ be the correspondence such that for each $\omega \in \Omega$, $h \circ F(\omega)=$ $h(F(\omega))$. Then $h \circ F$ is still measurable (see Proposition 2 on p. 59 of [20]). By the Filippov theorem (see [4, p. 316]), we know that any measurable selection of the correspondence $h \circ F$ is the composition of $h$ with a measurable selection of $F$. Hence $h\left(\int_{\Omega} F d L(P)\right)=\int_{\Omega} h \circ F d L(P)$. Since the linear function $h$ is continuous and $h \circ F$ is compact valued in $\mathbb{R}^{n}$, it can be checked that for any $\omega \in \Omega, h\left(\mathrm{w}^{*}-\overline{c o} F(\omega)\right)=$ $\overline{c o} h(F(\omega))=c o(h(F(\omega))$. Thus by Aumann's Theorem 3 in [6] (see also Theorem 4 on p. 64 of [20]), we have

$$
h\left(y_{0}\right)=\int_{\Omega} h(f) d L(P) \in \int_{\Omega} c o(h \circ F) d L(P)=\int_{\Omega}(h \circ F) d L(P)=h\left(\int_{\Omega} F d L(P)\right),
$$

which is a contradiction.

Proof of Theorem 10. For each $r>0$, let $B_{r}$ be the closed ball with center 0 and radius $r$ in $X^{*}$; then $B_{r}$ is weakly* compact. Let $M=\int_{\Omega} g d L(P)$. Then it follows from Theorem 8 that $\int_{\Omega} F(\cdot, y) d L(P)$ induces a closed valued correspondence from $Y$ to the compact space $B_{M}$. Let $\left\{y_{n}\right\}_{n=0}^{\infty}$ be a sequence in $Y$ with $\lim _{n \rightarrow \infty} y_{n}=y_{0}$. We first consider upper semicontinuity. Let $\left\{f_{n}\right\}_{n=1}^{\infty}$ be a sequence of Gel'fand integrable functions with $f_{n}$ a selection of $F\left(\cdot, y_{n}\right)$. Assume that $\lim _{n \rightarrow \infty} \int_{\Omega} f_{n} d L(P)=x_{0}$ in the weak* topology for some $x_{0} \in X^{*}$. The arguments used in the proof of Theorem 5 indicate that we only have to prove $x_{0} \in \int_{\Omega} F\left(\cdot, y_{0}\right) d L(P)$. It is clear that $B_{g(\cdot)}$ defines a compact valued correspondence from $(\Omega, L(\mathcal{A}), L(P))$ to the Polish space $\left(Z, d_{w}\right)$, which also dominates all the $F\left(\cdot, y_{n}\right)$ 's. For each $\omega \in \Omega$, since $F_{n}(\omega)$ 's are subsets of the weak* compact set $B_{g(\omega)}$, we know that the topological limit superior of the sequence $\left\{F\left(\omega, y_{n}\right)\right\}_{n=1}^{\infty}$ in $\left(Z, d_{w}\right)$ is still the same as $\mathrm{w}^{*}-\lim _{n \rightarrow \infty} \sup F\left(\omega, y_{n}\right)$ in $X^{*}$, which is a subset of $F\left(\omega, y_{0}\right)$ by the upper semicontinuity of $F(\omega, y)$ at $y_{0}$. By Proposition 3.4, we have $\lim _{n \rightarrow \infty} \sup \mathcal{D}_{F\left(\cdot, y_{n}\right)} \subseteq \mathcal{D}_{F\left(\cdot, y_{0}\right)}$ in the space of probability measures on $\left(Z, d_{w}\right)$ with the topology of weak convergence of measures. Thus there is a subsequence $\left\{f_{n_{k}}\right\}_{k=1}^{\infty}$ and a measurable selection $f$ of $F\left(\cdot, y_{0}\right)$ such that $\left\{L(P) f_{n_{k}}^{-1}\right\}_{k=1}^{\infty}$ converges weakly to $L(P) f^{-1}$. The integrable boundedness of $F$ indicates that $f$ is Gel'fand integrable. As in the proof of Theorem 3 , for each $m \geq 1, x_{m}^{* *}$ is uniformly continuous on $X^{*}$. Then we can 
obtain

$$
\lim _{k \rightarrow \infty} \int_{\Omega} x_{m}^{* *} \circ f_{n_{k}} d L(P)=\int_{\Omega} x_{m}^{* *} \circ f d L(P) .
$$

Since the $x_{m}^{* *}$ 's separate points in $X^{*}$, we have

$$
x_{0}=\int_{\Omega} f d L(P) \in \int_{\Omega} F\left(\cdot, y_{0}\right) d L(P),
$$

and thus the result on the preservation of weak* upper semicontinuity by integration is shown.

We can prove the result on the preservation of lower semicontinuity by using the characterization of lower semicontinuity by sequences (see [20, p. 27]) and by minor changes of the arguments used in the proof of Proposition 4.2.

Remark. If $X^{*}$ is also norm separable, then the Borel $\sigma$-algebras generated by the norm topology, the weak topology, and the weak* topology are the same. Any integrably bounded, weak* measurable function is also Bochner integrable. Thus, in this case, we can replace the Gel'fand integrals in Theorems 7-10 by Bochner integrals.

\section{REFERENCES}

1. S. Albeverio, J. E. Fenstad, R. Hoegh-Krohn, and T. L. Lindstrom, Nonstandard Methods in Stochastic Analysis and Mathematical Physics, Academic Press, Orlando, Florida, 1986. MR 88f:03061

2. R. M. Anderson, Star-finite representations of measure spaces, Transactions of the American Mathematical Society 271 (1982), 667-687. MR 83m:03077

3. Z. Artstein, Distributions of random sets and random selections, Israel Journal of Mathematics 46 (1983), 313-324. MR 85m:60017

4. J.-P. Aubin and H. Frankowska, Set Valued Analysis, Birkhäuser, Boston, 1990. MR 91d:49001

5. R. J. Aumann, Markets with a continuum of traders, Econometrica 32 (1964), 39-50. MR 30:2908

6. Integrals of set valued functions, Journal of Mathematical Analysis and Applications 12 (1965), 1-12. MR 32:2543

7. E. J. Balder, Fatou's lemma in infinite dimensions, Journal of Mathematical Analysis and Applications 136 (1988), 450-465. MR 90e:28012

8. C. Berge, Topological Spaces, Oliver \& Boyd, London, 1959. MR 21:4401 (French ed.)

9. P. Billingsley, Convergence of Probability Measures, Wiley, New York, 1968. MR 38:1718

10. C. L. Byrne, Remarks on the set-valued integrals of Debreu and Aumann, Journal of Mathematical Analysis and Applications 62 (1978), 243-246. MR 80a:28006

11. C. Castaing and M. Valadier, Convex Analysis and Measurable Multifunctions, Lecture Notes in Mathematics, vol. 580, Springer-Verlag, Berlin and New York, 1977. MR 57:7169

12. G. Debreu, Integration of correspondences, Proceedings of the Fifth Berkeley Symposium on Mathematical Statistics and Probability, vol. 2, part 1, Univ. of California Press, 1967, pp. 351-372. MR 37:3835

13. J. Diestel and J. J. Uhl, Vector Measures, Mathematical Surveys, vol. 15, American Mathematical Society, Providence, RI, 1977. MR 56:12216

14. N. Dunford and J. Schwartz, Linear Operators, Part I, Interscience, New York, 1958. MR 22:8302

15. Ky Fan, Fixed-point and minimax theorems in locally convex linear spaces, Proceedings of the National Academy of Sciences of the USA 38 (1952), 121-126. MR 13:858d

16. I. Glicksberg, A further generalization of Kakutani's fixed point theorem with application to Nash equilibrium points, Proceedings of the American Mathematical Society 3 (1952), 170174. MR 13:764g 
17. S. Hart, W. Hildenbrand, and E. Kohlberg, On equilibrium allocations as distributions on the commodity space, Journal of Mathematical Economics 1 (1974), 159-166. MR 55:9863

18. S. Hart and E. Kohlberg, Equally distributed correspondences, Journal of Mathematical Economics 1 (1974), 167-174. MR 54:10540

19. F. Hiai and H. Umegaki, Integrals, conditional expectations, and martingales of multivalued functions, Journal of Multivariate Analysis 7 (1977), 149-182. MR 58:22463

20. W. Hildenbrand, Core and Equilibria of a Large Economy, Princeton University Press, Princeton, 1974. MR 52:9991

21. R. B. Holmes, Geometric Functional Analysis and Its Applications, Springer-Verlag, New York, 1975. MR 53:10485

22. A. E. Hurd and P. A. Loeb, An Introduction to Nonstandard Real Analysis, Academic Press, Orlando, Florida, 1985. MR 87d:03184

23. R. James, Weakly compact sets, Transactions of the American Mathematical Society 113 (1964), 129-140. MR 29:2628

24. $\ldots$ A counterexample for a sup theorem in normed spaces, Israel Journal of Mathematics 9 (1971), 511-512. MR 43:5287

25. M. A. Khan, On the integration of set-valued mappings in a non-reflexive Banach space, II, Simon Stevin 59 (1985), 257-267. MR 87g:28009

26. M. A. Khan and M. Majumdar, Weak sequential convergence in $L_{1}(\mu, X)$ and an approximate version of Fatou's lemma, Journal of Mathematical Analysis and Applications 114 (1986), 569-573. MR 87m:28004

27. M. A. Khan and Y. N. Sun, Non-cooperative games on hyperfinite Loeb spaces, submitted.

28. M. A. Khan and Y. N. Sun, General equilibrium theory with a Loeb space of agents, presented at the Workshop on Geometry, Topology and Markets at the Fields Institute for Research in Mathematical Sciences in July 1994.

29. E. Klein and A. C. Thompson, Theory of Correspondences, Wiley, New York, 1984. MR 86a:90012

30. G. Knowles, Lyapunov vector measures, SIAM J. Control 13 (1975), 294-303. MR 52:9053

31. P. A. Loeb, Conversion from nonstandard to standard measure spaces and applications in probability theory, Transactions of the American Mathematical Society 211 (1975), 113-122. MR 52:10980

32. C. Olech, Existence theory in optimal control, Control Theory and Topics in Functional Analysis, International Atomic Energy Agency, Vienna, 1976, pp. 291-328. MR 58:23858

33. H. Osswald and Y. N. Sun, On the extensions of vector-valued Loeb measures, Proceedings of the American Mathematical Society 111 (1991), 663-675. MR 92g:28041

34. K. R. Parthasarathy, Probability Measures on Metric Spaces, Academic Press, New York, 1967. MR 37:2271

35. P. Pucci and G. Vitillaro, A representation theorem for Aumann integrals, Journal of Mathematical Analysis and Applications 102 (1984), 86-101. MR 86d:28016

36. S. Rashid, Economies with Many Agents: An Approach Using Nonstandard Analysis, Johns Hopkins University, Baltimore, 1987. MR 90h:90041

37. H. Richter, Verallgemeinerung eines in der Statictik benötigten Satzes der Masstheorie, Mathematische Annalen 150 (1963), 85-90. MR 26:3851

38. A. Rustichini, A counterexample and an exact version of Fatou's lemma in infinite dimensional spaces, Archiv der Mathematik 52 (1989), 357-362. MR 90k:46101

39. A. Rustichini and N. Yannelis, What is perfection competition?, Equilibrium Theory in Infinite Dimensional Spaces (M. A. Khan and N. C. Yannelis, eds.), Springer-Verlag, Berlin, 1991.

40. D. Schmeidler, Fatou's lemma in several dimensions, Proceedings of the American Mathematical Society 24 (1970), 300-306. MR 40:1568

41. Y. N. Sun, Nonstandard theory of vector measures, Ph.D. dissertation, University of Illinois, Urbana, Illinois, 1989.

42. - On the theory of vector valued Loeb measures and integration, Journal of Functional Analysis 104 (1992), 327-362. MR 93a:46146

43. _ Distributional properties of correspondences on Loeb spaces, Journal of Functional Analysis 139 (1996), 68-93. CMP 96:15

44. D. H. Wagner, Survey of measurable selection theorems, SIAM J. Control and Optimization 15 (1977), 859-903. MR 58:6137 
45. N. C. Yannelis, On the upper and lower semicontinuity of the Aumann integral, Journal of Mathematical Economics 19 (1990), 373-389. MR 92d:26021

46. _ Integration of Banach-valued correspondences, Equilibrium Theory in Infinite Dimensional Spaces (M. A. Khan and N. C. Yannelis, eds.), Springer-Verlag, Berlin, 1991.

Department of Mathematics, National University of Singapore, Singapore 119260

Current address: Cowles Foundation, Yale University, New Haven, Connecticut 06520

E-mail address: gs53@econ.yale.edu 